\title{
Binary mixtures of rod-like colloids under shear: microscopically-based equilibrium theory and order-parameter dynamics
}

\author{
Rodrigo Lugo-Frías and Sabine H. L. Klapp \\ Institute für Theoretische Physik, Technische Universität Berlin, Hardenbergstrasse \\ 36, 10623 Berlin, Germany \\ E-mail: klapp@physik.tu-berlin.de
}

\begin{abstract}
This paper is concerned with the dynamics of a binary mixture of rodlike, repulsive colloidal particles driven out of equilibrium by means of a steady shear flow (Couette geometry). To this end we first derive, starting from a microscopic density functional in Parsons-Lee approximation, a mesoscopic free energy functional whose main variables are the orientational order parameter tensors. Based on this mesoscopic functional we then explore the stability of isotropic and nematic equilibrium phases in terms of composition and rod lengths. Second, by combining the equilibrium theory with the Doi-Hess approach for the order parameter dynamics under shear, we investigate the orientational dynamics of binary mixtures for a range of shear rates and coupling parameters. We find a variety of dynamical states, including synchronized oscillatory states of the two components, but also symmetry breaking behavior where the components display different in-plane oscillatory states.
\end{abstract}

Keywords: density functional theory, Q-tensor, rod-like colloids, binary mixtures, shearinduced dynamics

\section{Introduction}

Mixtures of rod-like colloidal particles are found in many natural systems, e.g., in cells and in the blood, as well as in various industrial applications such as paints and in food science. Typically, these colloidal systems are dominated by the repulsive, anisotropic interactions stemming from the particle's non-spherical shape. Already in thermodynamic equilibrium, such mixtures display a very rich, entropy-driven phase behavior 1, 2, 3, 4, 5, characterized by the interplay of orientational ordering, (depletion-induced) condensation and demixing. A recent example is an experimental study of polydisperse clay rods which show an isotropic-nematic phase transition on increasing concentration [6]. From the theoretical side, such phase diagrams have been studied, e.g., by Onsager theory [7] and by more general free energy functionals [8, 9, 10] 
in the framework of classical density functional theory (DFT), as well as by computer simulations [11, 12].

While the equilibrium behavior is already challenging, a further level of complexity is reached when considering such mixtures under shear flow [13, 14, 15]. The current interest in this topic has basically two reasons: first, investigating the impact of (shear-) flow is crucial for understanding the system's rheological behavior, an aspect which is particularly important from the application side. Second, shear flow is a fundamental example of driving a soft-matter system out of equilibrium. Indeed, already for onecomponent systems of colloidal rods, shear can have profound consequences. Examples are the shear-induced shift of the isotropic-nematic transition and the associated stabilization of the nematic phase [16, 17], and the occurrence of spatial symmetry breaking related to shear-banding [18, 19, 20]. Moreover, even under steady shear the rod-like particles can display spontaneous time-dependent oscillations, a prime example being the tumbling motion (characterized by full rotations of the particle axes). Indeed, tumbling has been observed in experiments [21] as well as in particle-resolved computer simulations [22, 23].

Another well-established route to explore the orientational dynamics under shear are mesoscopic (continuum) equations, such as the Doi-Hess approach [24, 25, 26, 27, 28, 29], describing the evolution of an appropriate order parameter in time. For one-component systems of rods with head-tail symmetry, this mesoscopic order parameter is the so-called Q-tensor, a second-rank tensor which corresponds to the lowest non-vanishing moment of the one-body orientational distribution function $f=f(\boldsymbol{\omega})$ (where $\boldsymbol{\omega}=(\theta, \phi)$ contains the set of Euler angles describing the particle's orientation [30, 31]). Within the Doi-Hess approach [26, 27, 28], the time dependence of $\mathrm{Q}$ is described by a competition between flow-induced perturbations, on the one hand, and a relaxational contribution stemming from the (functional) derivative of the phenomenological Landau-de Gennes (LdG) free energy [30], on the other hand. The underlying equilibrium behavior is then determined by the coefficients of the powers of the order parameter in the LdG free energy. Usually, these coefficients are constants which do not depend on a specific microscopic Hamiltonian, and are thus, independent of e.g., the aspect ratio of the particles; rather one uses the LdG functional as an universal free energy expression yielding an isotropic-nematic (I-N) transition. The resulting, nonlinear equations of motion indeed generate complex dynamical behavior characterized by different (regular) oscillatory states [32, 33, 34, chaotic behavior [35] and spatio-temporal patterns [36, 37].

In the present paper we employ mesoscopic equations inspired by the Doi-Hess approach to investigate the shear-induced dynamics of binary rod mixtures. However, we modify the original approach by replacing the standard LdG free energy functional (for mixtures) by a free energy functional derived from classical DFT. In this way, our theory incorporates microscopic information, particularly the aspect ratios and the molar fractions characterizing the two species in the mixtures.

Classical DFT is a powerful tool to study inhomogeneous fluids near interfaces and 
in other external potentials [38, 39] and has nowadays a wide range of applications both for sphere-like systems and for systems of shape-anisotropic particles. The key idea of DFT is to minimize a (free energy) functional of the singlet density $\rho(\mathbf{r}, \boldsymbol{\omega})$ [10, 40]. Several studies have been devoted to construct free energy functionals for homogeneous states of anisotropic fluids, such that $\rho(\mathbf{r}, \boldsymbol{\omega})=\rho f(\boldsymbol{\omega})$ (where $\rho=N / V$ is the number density). Here one main goal was to describe the I-N phase transition [41, 42]. Of special importance for the present study is the work of Malijevsky et al. [43], who developed a DFT for the description of spatial homogeneous phases (isotropic and nematic) of hard-body fluid mixtures by generalizing the ideas of Parsons [8] and Lee [9] for pure component systems.

Here we use this multicomponent Parsons-Lee theory [43] as a starting point to construct an (approximate) free energy functional containing powers of the tensorial order parameters $\mathbf{Q}^{i}$, where $i=A, B$ labels the two components of the mixture. The coefficients in this expansion contain the microscopic information mentioned before. We then combine our equilibrium theory with a straightforward extension of the dynamical Doi-Hess equations for a binary mixture to study the full order parameter dynamics of the system under (Couette) shear flow. By combining microscopic (DFT) and mesoscopic "ingredients", we consider our approach as a contribution to a scale-bridging description of complex colloidal mixtures far from equilibrium. To some extent, our work is related to recent derivations of phase-field-crystal models (which include gradient terms) starting from microscopic functionals [44, 45].

The paper is organized as follows. In section 2 we first define in detail the hardbody binary mixture and present the microscopic (Parsons-Lee) free energy functional. We then perform an expansion into Q-tensors, yielding a "mesoscopic" functional representing the orientational part of the full free energy up to fourth order in the order parameters. In sections 2.3 and 2.4, we then specialize to mixtures of hard spherocylinders in the semi-dilute regime and investigate the stability of isotropic and nematic states (for fixed densities). Section 3.1 is devoted to the explicit equations of motion in the framework of the Doi-Hess theory. In section 3.3. we present numerical results including full non-equilibrium state diagrams portraying the dynamic behavior of the orientational order parameters. The paper finishes with concluding remarks and an outlook in section 4 . Some technical details are given in an appendix.

\section{Equilibrium Theory}

We consider a binary mixture of hard, uniaxial particles with repulsive pair interactions dependent on their orientations. The two species are labeled by indices $i, j$, which take values $A$ or $B$. The pair potential between a particle " 1 " of component $i$ and a particle " 2 " of component $j$ is then given by (see, e.g., [43])

$$
u_{i j}\left(\boldsymbol{r}_{12}, \boldsymbol{\omega}_{1}, \boldsymbol{\omega}_{2}\right)= \begin{cases}\infty & r_{12}<d_{i j}\left(\boldsymbol{\omega}_{1}, \boldsymbol{\omega}_{2}, \boldsymbol{\omega}_{12}\right) \\ 0 & \text { otherwise }\end{cases}
$$


where $d_{i j}\left(\boldsymbol{\omega}_{1}, \boldsymbol{\omega}_{2}, \boldsymbol{\omega}_{12}\right)$ is the orientational dependent distance of closest approach of the two particles, $r_{12}$ is the magnitude of the center-to-center vector $\mathbf{r}_{12}=r_{12} \boldsymbol{\omega}_{12}$, $\boldsymbol{\omega}_{1}=\left(\theta_{1}, \varphi_{1}\right)$ and $\boldsymbol{\omega}_{2}=\left(\theta_{2}, \varphi_{2}\right)$ are the Euler angles of the two particles, and $\boldsymbol{\omega}_{12}=\left(\theta_{12}, \varphi_{12}\right)$ describes the orientation of $\mathbf{r}_{12}$.

\subsection{Microscopic free energy functional}

Within the formalism of classical DFT, the total Helmholtz free energy is written as a sum of ideal and excess contributions, such that $\mathcal{F}=\mathcal{F}_{i d}+\mathcal{F}_{\text {ex }}$ [38, 39]. This quantity is a functional of the one-particle densities $\rho_{i}(\boldsymbol{r}, \boldsymbol{\omega})$. Here we focus on spatial homogeneous phases such that $\rho_{i}(\boldsymbol{r}, \boldsymbol{\omega})=\rho_{i} f_{i}(\boldsymbol{\omega})$, where $\rho_{i}=N_{i} / V$ is the number density of species $i$ (with $V$ being the volume) and $f_{i}(\boldsymbol{\omega})$ is the orientational distribution function (ODF), which is normalized such that $\int d \boldsymbol{\omega} f_{i}(\boldsymbol{\omega})=1$. This ansatz allows us to describe isotropic and nematic phases of the system.

With this specification, the ideal part of the free energy functional can be written as

$$
\frac{\beta \mathcal{F}_{i d}}{V}\left[\left\{\rho_{i}\right\}\right]=\sum_{i=A, B} \rho_{i}\left(\ln \left(\rho_{i} \Lambda_{i}^{3}\right)-1+\sigma\left[f_{i}\right]\right)
$$

where $\beta=1 / k_{B} T$ ( $T$ being the temperature and $k_{B}$ Boltzmann's constant), $\Lambda_{i}$ is the thermal de Broglie wavelength of each component, and $\sigma\left[f_{i}\right]$ is the orientational entropy given by

$$
\sigma\left[f_{i}\right]=\int d \boldsymbol{\omega} f_{i}(\boldsymbol{\omega}) \ln \left(4 \pi f_{i}(\boldsymbol{\omega})\right)
$$

where $\int d \boldsymbol{\omega}=\int_{-1}^{1} d \cos \theta \int_{0}^{2 \pi} d \varphi$. The quantity $\sigma_{i}$ is zero in the isotropic state, where $f_{i}(\boldsymbol{\omega})=f_{0}=(4 \pi)^{-1}$ and becomes positive in the nematic state. It thus reflects the loss of orientational entropy due to ordering.

Regarding the excess part of the free energy, we here employ a generalization of the so-called Parsons-Lee theory [8, 9] to mixtures, as suggested by Malijevsky et al. [43]. Briefly, this approach is derived by starting from the virial expression for the pressure, which involves (apart from the singlet densities) the spatial derivative of the pair potential, $u_{i j}$ and the pair correlation function $g_{i j}$. Noting that $u_{i j}$ can be re-expressed in terms of a hard-sphere (HS) potential with scaled distance $r_{12} / d_{i j}\left(\boldsymbol{\omega}_{1}, \boldsymbol{\omega}_{2}, \boldsymbol{\omega}_{12}\right)$, the same assumption is made for the correlation function, i.e., $g_{i j}=g_{i j}^{H S}\left(r_{12} / d_{i j}\right)$ (note that this is an approximation). By integrating over the total density $\rho=\sum_{i} \rho_{i}$ (and noting that $\partial u_{i j}^{H S}(y) / \partial y$ is proportional to a delta function in space), one obtains the excess free energy

$$
\frac{\beta \mathcal{F}_{e x}}{V}\left[\left\{\rho_{i}\right\}\right]=\frac{1}{2} \sum_{i=A, B} \sum_{j=A, B} \rho_{i} \rho_{j} G_{i j} \int d \boldsymbol{\omega}_{1} \int d \boldsymbol{\omega}_{2} V_{i j}^{e x c}\left(\boldsymbol{\omega}_{1}, \boldsymbol{\omega}_{2}\right) f_{i}\left(\boldsymbol{\omega}_{1}\right) f_{j}\left(\boldsymbol{\omega}_{2}\right) .
$$

In (41), $V_{i j}^{e x c}$ is the excluded volume between particles 1 and 2 , which is related to the contact distance via

$$
V_{i j}^{e x c}\left(\boldsymbol{\omega}_{1}, \boldsymbol{\omega}_{2}\right)=\frac{1}{3} \int d \boldsymbol{\omega}_{12} d_{i j}^{3}\left(\boldsymbol{\omega}_{1}, \boldsymbol{\omega}_{2}, \boldsymbol{\omega}_{12}\right) .
$$


Further, the quantities $G_{i j}$ are averages (in terms of the total density) of the contact values of the HS pair correlation functions, $g_{i j}^{H S}\left(1_{+}\right)$, in the considered density range.

As discussed in [43], the free energy given in (44) reduces to the more familiar second-virial theory of Onsager [7] in the limit of low densities. Then, one has

$g_{i j}^{H S}\left(1_{+}\right) \rightarrow 1$, and therefore $G_{i j}=1$ for all combinations $i, j$. Although the Onsager theory provides an intuitive approach regarding the effects related to the geometry of the particles, it is known to become inaccurate for particles of finite length at the densities close to orientational phase transitions [11, 12]. In this respect the generalized ParsonsLee approach provides a significant improvement, as demonstrated by comparison with Monte Carlo simulations of hard Gaussian molecules with different elongations and concentrations [46].

\subsection{Mesoscopic free energy functional}

The aim of this section is to rewrite the microscopic free energy functional consisting of the ideal and excess parts given in equations (2) and (4), respectively, in terms of "mesoscopic" orientational order parameters, specifically, the tensors, $\mathbf{Q}_{(k)}^{i}, i=A, B$, of rank $k$. These tensors are defined as [31]

$$
\mathbf{Q}_{(k)}^{i}=\sqrt{\frac{(2 k+1) ! !}{k !}}\left\langle\overline{\boldsymbol{\omega}^{(k)}}\right\rangle_{i},
$$

where $\boldsymbol{\omega}$ is a unit vector with the orientation $\boldsymbol{\omega}=(\theta, \varphi),\langle\cdots\rangle_{i}=\int d \boldsymbol{\omega}_{i} f\left(\boldsymbol{\omega}_{i}\right) \cdots$ is an average involving the orientational distribution function, and the notation $\overrightarrow{\mathbf{A}}$ denotes the symmetric traceless part of the tensor $\mathbf{A}$. Here, $\mathbf{A}=\boldsymbol{\omega}^{(k)}$ is a $k^{-}$ fold tensorial product of the form $\boldsymbol{\omega} \otimes \cdots \otimes \boldsymbol{\omega}$ (e.g., for the case $k=2$ this yields $\left.\left(\boldsymbol{\omega}^{(2)}\right)_{m n}=(\boldsymbol{\omega})_{m}(\boldsymbol{\omega})_{n}\right)$.

For the present systems, which involve uniaxial particles with head-tail symmetry, it is sufficient to consider tensors with even $k$. Within this subset, the tensors of rank $k=2$ and $k=4$ are of particular importance since they are related to physical phenomena such as birefringence and fluorescence [30, 29]. Tensors with odd values of $k$ occur in the description of systems with additional degrees of freedom such as rod-like ferrofluids [47, 48].

2.2.1. Ideal contribution The first task is to rewrite the orientational entropy, given in (3). Here we follow earlier approaches [29, 48], where the one-particle ODF was expressed as $f_{i}(\boldsymbol{\omega})=f_{0}\left(1+\alpha_{i}(\boldsymbol{\omega})\right)$, with $\alpha(\boldsymbol{\omega})$ being a small deviation from the isotropic state, $f_{0}=1 / 4 \pi$. Inserting this ansatz into (3) and performing a Taylor expansion of the logarithm yields [29, 49]

$$
\sigma\left[f_{i}\right]=\sum_{n=2}^{\infty} \frac{(-1)^{n}}{n(n-1)}\left\langle\alpha_{i}^{n}(\boldsymbol{\omega})\right\rangle_{0},
$$

where $\langle\cdots\rangle_{0}$ denotes the orientational average evaluated with $f_{0}$. Due to normalization of the ODF the term $n=1$ is zero and thus it is disregarded. Next, one assumes that the 
function $\alpha(\boldsymbol{\omega})$ can be expanded in terms of the tensorial quantities $( ( 2 k + 1 ) ! ! / k ! ) ~ \longdiv { \boldsymbol { \omega } ^ { ( k ) } }$ [see (6)], where the prefactors are determined by their orientational averages, $\mathbf{Q}_{(k)}^{i}$. Following [29, 49], we restrict this expansion to terms involving $k=2$ and $k=4$. Inserting the resulting ansatz for $\alpha_{i}$ into (7) and retaining terms up to fourth power in $\mathbf{Q}_{(2)}^{i}$ and up to second power in $\mathbf{Q}_{(4)}^{i}$, one obtains

$$
\begin{array}{r}
\sigma\left(\mathbf{Q}_{(2)}^{i}, \mathbf{Q}_{(4)}^{i}\right)=\frac{1}{2} \mathbf{Q}_{(2)}^{i} \odot \mathbf{Q}_{(2)}^{i}-\frac{\sqrt{30}}{21} \mathbf{Q}_{(2)}^{i} \cdot \mathbf{Q}_{(2)}^{i} \odot \mathbf{Q}_{(2)}^{i} \\
+\frac{5}{28}\left[\mathbf{Q}_{(2)}^{i} \odot \mathbf{Q}_{(2)}^{i}\right]^{2}+\frac{1}{2} \mathbf{Q}_{(4)}^{i} \odot \mathbf{Q}_{(4)}^{i} .
\end{array}
$$

In (8)), the product $\mathbf{A}_{(k)} \cdot \mathbf{B}_{(k)}$ yields a tensor of rank $k$ (with components $A_{i j \ldots m n} B_{n m \ldots k l}=$ $C_{i j \ldots k l}$ (where we used the Einstein convention). Further, $\mathbf{A}_{(k)} \odot \mathbf{B}_{(k)}=A_{i j \ldots k l} B_{i j \ldots k l}=c$ indicates the maximal possible contraction, that is, $c$ is a scalar.

2.2.2. Excess contribution We now turn to the reformulation of the excess free energy as functions of the $\mathbf{Q}_{(k)}^{i}$. To our knowledge, this problem has not been addressed in the literature so far. As a starting point we expand the excluded volume of the hard-core particles appearing in (4) in terms of the Legendre polynomials (following [43, 50, and references therein),

$$
V_{i j}^{e x c}\left(\boldsymbol{\omega}_{1}, \boldsymbol{\omega}_{2}\right)=\sum_{k=0}^{\infty} V_{k}^{i j} P_{k}(\cos \gamma),
$$

where $\gamma$ is the angle between $\boldsymbol{\omega}_{1}$ and $\boldsymbol{\omega}_{2}$, and the coefficients $V_{k}^{i j}$ are calculated from the relation

$$
V_{k}^{i j}=\frac{2 k+1}{2} \int_{-1}^{1} d \cos \gamma P_{k}(\cos \gamma) V_{i j}^{e x c}(\gamma) .
$$

Next, we replace $P_{k}$ in (9) by using the addition theorem for spherical harmonics [51], yielding

$$
V_{i j}^{e x c}\left(\boldsymbol{\omega}_{1}, \boldsymbol{\omega}_{2}\right)=\sum_{k=0}^{\infty} \frac{4 \pi V_{k}^{i j}}{2 k+1} \sum_{m=-k}^{k} Y_{k m}^{*}\left(\boldsymbol{\omega}_{1}\right) Y_{k m}\left(\boldsymbol{\omega}_{2}\right) .
$$

The sum on the right side of (11) can be rewritten as a full scalar product of the two tensors $\longdiv { \boldsymbol { \omega } ^ { ( k ) } }$ [51]. One obtains

$$
V_{i j}^{e x c}\left(\boldsymbol{\omega}_{1}, \boldsymbol{\omega}_{2}\right)=\sum_{k=0}^{\infty} V_{k}^{i j} \frac{(2 k+1) ! !}{(2 k+1) k !} \underset{\boldsymbol{\omega}_{1}^{(k)}}{ } \odot \overrightarrow{\boldsymbol{\omega}_{2}^{(k)}} .
$$

Inserting (12) into (4), noting that the angular integrals (weighted by the ODFs) in (4) lead to orientational averages, and using the definition of the tensorial order parameter [see (6) $]$ one finds

$$
\frac{\beta \mathcal{F}_{\text {exc }}}{V}=\frac{1}{2} \sum_{i, j} \rho_{i} \rho_{j} G_{i j} \sum_{k=0}^{\infty} \frac{V_{k}^{i j}}{2 k+1} \mathbf{Q}_{(k)}^{i} \odot \mathbf{Q}_{(k)}^{j} .
$$


Equation (13) provides the excess free energy as a function of order parameter tensors. It still depends on the microscopic parameters of the mixture, that is, the geometry of the particles (defining the excluded volume) and the densities.

2.2.3. Full free energy We now turn to the full free energy containing ideal and excess parts, $\mathcal{F}=\mathcal{F}_{i d}+\mathcal{F}_{e x}$. Restricting to terms involving $2 n d-$ and $4 t h-$ rank tensors we have

$$
\frac{\beta F_{i d}[\{\mathbf{Q}\}]}{V}=\sum_{i=A, B}\left[\mathcal{F}_{00}^{i}+\rho_{i} \sigma\left(\mathbf{Q}_{(2)}^{i}, \mathbf{Q}_{(4)}^{i}\right)\right],
$$

where $\mathcal{F}_{00}^{i}=\rho_{i}\left(\ln \left(\rho_{i} \Lambda_{i}\right)-1\right), \sigma$ is given in (8) , and the notation $[\{\mathbf{Q}\}]$ on the left side indicates the dependence of the (ideal) free energy on all four order parameter tensors $(k=2,4, i=A, B)$. Further, we obtain from (13)

$$
\frac{\beta \mathcal{F}_{e x}[\{\mathbf{Q}\}]}{V}=\frac{1}{2} \sum_{i, j} \rho_{i} \rho_{j} G_{i j}\left[V_{0}^{i j}+\frac{V_{2}^{i j}}{5}\left(\mathbf{Q}_{(2)}^{i} \odot \mathbf{Q}_{(2)}^{j}\right)+\frac{V_{4}^{i j}}{9}\left(\mathbf{Q}_{(4)}^{i} \odot \mathbf{Q}_{(4)}^{j}\right)\right] .
$$

These expressions can be further simplified by using a "closure" relation expressing the $4 t h$-rank tensor with the one of second rank. For uniaxial systems characterized by a (nematic) director $\mathbf{n}$ [47, 52], there exists the exact relation $S_{2}^{2} \mathbf{Q}_{(4)}=S_{4} \overline{\mathbf{Q}_{(2)} \mathbf{Q}_{(2)}}$, where $S_{2}=\left\langle P_{2}(\boldsymbol{\omega} \cdot \mathbf{n})\right\rangle$ and $S_{4}=\left\langle P_{4}(\boldsymbol{\omega} \cdot \mathbf{n})\right\rangle$. In strongly aligned systems one has $S_{4} \approx S_{2}^{2} \approx 1$. In that limit, the closure thus takes the form $\mathbf{Q}_{(4)} \equiv \overline{\mathbf{Q}_{(2)} \mathbf{Q}_{(2)}}$.

Since we can now express all quantities in terms of the second-rank tensor, we ease notation and set $\mathbf{Q}^{i}=\mathbf{Q}_{(2)}^{i}(i=A, B)$. Further, we rewrite the notation for the tensor products appearing in (15) into the form commonly used for second-rank tensors, that is $\mathbf{Q}^{i} \odot \mathbf{Q}^{i}=\mathbf{Q}^{i}: \mathbf{Q}^{i}=\operatorname{Tr}(\mathbf{Q} \cdot \mathbf{Q})=\sum_{m n} Q_{m n} Q_{m n}$. Adding ideal and excess contributions of the free energy, we obtain

$$
\begin{aligned}
& \frac{\beta \mathcal{F}(\{\mathbf{Q}\})}{V}=\sum_{i=A, B}\left.\left\{\mathcal{F}_{0}^{i}+A_{i}\left(\mathbf{Q}^{i}: \mathbf{Q}^{i}\right)-B_{i} \operatorname{Tr}\left(\mathbf{Q}^{i} \cdot \mathbf{Q}^{i} \cdot \mathbf{Q}^{i}\right)+C_{i}\left(\mathbf{Q}^{i}: \mathbf{Q}^{i}\right)^{2}\right)\right\} \\
&+\mathcal{F}_{0}^{A B}+A_{A B}\left(\mathbf{Q}^{A}: \mathbf{Q}^{B}\right)+C_{A B}\left(\mathbf{Q}^{A}: \mathbf{Q}^{B}\right)^{2},
\end{aligned}
$$

where the coefficients are functions of the number densities, the factors $G_{i j}$ and the coefficients of the excluded volume. Explicitly, they are given by

$$
\begin{aligned}
\mathcal{F}_{0}^{i} & =\rho_{i}\left(\ln \left(\rho_{i} \Lambda_{i}\right)-1\right)+\frac{1}{2} \rho_{i}^{2} G_{i i} V_{0}^{i i}, \\
A_{i} & =\frac{1}{2} \rho_{i}+\frac{1}{10} \rho_{i}^{2} G_{i i} V_{2}^{i i}, \\
B_{i} & =\frac{\sqrt{30}}{21} \rho_{i}, \\
C_{i} & =\frac{19}{28} \rho_{i}+\frac{1}{18} \rho_{i}^{2} G_{i i} V_{4}^{i i}, \\
\mathcal{F}_{0}^{A B} & =\rho_{A} \rho_{B} G_{A B} V_{0}^{A B}, \\
A_{A B} & =\frac{1}{5} \rho_{A} \rho_{B} G_{A B} V_{2}^{A B}, \\
C_{A B} & =\frac{1}{9} \rho_{A} \rho_{B} G_{A B} V_{4}^{A B} .
\end{aligned}
$$


As seen from (16), the "mesoscopic" free energy derived here has the standard Landaude Gennes (LdG) form [30] in the sense that it involves an expansion into order parameters. Contrary to standard LdG theory, however, the coefficients are related to microscopic properties of the system. We also note that the free energy does not depend explicitly on the temperature, which reflects the hard-core character of interactions. Thus, the ordering behavior of the resulting "lyotropic" system is essentially controlled by the concentration of the system.

\subsection{Specialization to mixtures of hard spherocylinders}

The derivation presented so far can be applied to binary mixtures of any hard-core particles which are uniaxial and have head-tail symmetry. In the following we specify on systems of hard spherocylinders. Hard spherocylinders are characterized by two geometrical parameters, their length $l_{i}$ and their diameter $d_{i}$, defining their aspect ratio $\kappa_{i}=l_{i} / d_{i}$. The resulting particle volume is $v_{i}=(\pi / 12)\left(3 \kappa_{i}+2\right) d_{i}^{3}$. The excluded volume $V_{\text {exc }}(\gamma)$ [defined in (5)] of two spherocylinders was calculated by Onsager in 1949 [7]; a modern derivation has been given in the framework of general expressions for excluded volumes between hard sphero-zonotopes (see [53]). The result is

$$
\begin{gathered}
V_{\text {exc }}(\gamma)=\frac{\pi}{4}\left(\kappa_{i} d_{i}^{3}+\kappa_{j} d_{j}^{3}\right)+\frac{\pi}{4} d_{i} d_{j}\left(d_{i}+d_{j}\right) \sin \gamma+\frac{\pi}{4}\left(\kappa_{i} d_{i} d_{j}^{2}+\kappa_{j} d_{j} d_{i}^{2}\right)|\cos \gamma| \\
+\kappa_{i} \kappa_{j} d_{i} d_{j}\left(d_{i}+d_{j}\right) \sin \gamma+\left(\kappa_{i} d_{i}+\kappa_{j} d_{j}\right) d_{i} d_{j} E(\sin \gamma),
\end{gathered}
$$

where $E(\sin \gamma)$ denotes the complete elliptic integral of the second kind. Within the original Onsager theory, only terms up to linear order in $\sin \gamma$ are considered; that is, the terms involving $|\cos \gamma|$ and $E(\sin \gamma)$ are neglected. In the present work we keep the full expression (18). To calculate the resulting expansion coefficients $V_{k}^{i j}$ which are defined in (10) and which determine the coefficients appearing in the free energy [see (17a $)-(17 g)]$, we perform a series expansion as described in Appendix A. In this way we obtain analytic expressions for the coefficients of orders $k=0,2,4$.

In the following we focus on the part of the free energy which depends on the orientational order parameters, that is,

$$
\beta \mathcal{F}^{\text {or }} / V=\beta \mathcal{F}(\{\mathbf{Q}\}) / V-\left(\mathcal{F}_{0}^{A}+\mathcal{F}_{0}^{B}+\mathcal{F}_{0}^{A B}\right),
$$

where the terms on the right side are defined in equations (16) and (17a) $-(17 b)$, respectively. We further specialize on situations where the hard spherocylinders have equal diameters, i.e., $d_{A}=d_{B}=d$, but different aspect ratios, $\kappa_{A} \neq \kappa_{B}$. This allows to introduce a dimensionless form of the orientational free energy, $\mathscr{F}^{\text {or }}=\beta \mathcal{F}^{\text {or }} d^{3} / V$, and the reduced densities $\rho_{i}^{*}=\rho_{i} d^{3}$.

With these assumptions, the orientational free energy of a binary mixture of hard spherocylinders becomes

$$
\begin{aligned}
\mathscr{F}^{o r}= & \mathscr{F}^{o r}\left(\left\{\rho_{i}^{*}, \kappa_{i}\right\} ;\left\{\mathbf{Q}^{i}\right\}\right) \\
= & \sum_{i=A, B}\left\{A_{i}\left(\rho_{i}^{*}, \kappa_{i}\right)\left(\mathbf{Q}^{i}: \mathbf{Q}^{i}\right)-B_{i}\left(\rho_{i}^{*}\right) \operatorname{Tr}\left(\mathbf{Q}^{i} \cdot \mathbf{Q}^{i} \cdot \mathbf{Q}^{i}\right)+C_{i}\left(\rho_{i}^{*}, \kappa_{i}\right)\left(\mathbf{Q}^{i}: \mathbf{Q}^{i}\right)^{2}\right\} \\
& \quad-A_{A B}\left(\left\{\rho_{i}^{*}\right\},\left\{\kappa_{i}\right\}\right)\left(\mathbf{Q}^{A}: \mathbf{Q}^{B}\right)-C_{A B}\left(\left\{\rho_{i}^{*}\right\},\left\{\kappa_{i}\right\}\right)\left(\mathbf{Q}^{A}: \mathbf{Q}^{B}\right)^{2},
\end{aligned}
$$


where the coefficients $A_{i}, B_{i}, C_{i}, A_{A B}$ and $C_{A B}$ are given by

$$
\begin{aligned}
A_{i}\left(\rho_{i}^{*}, \kappa_{i}\right) & =\frac{1}{2} \rho_{i}^{*}-\frac{1}{10} \rho_{i}^{* 2}\left(\frac{5 \pi^{2}}{64}-\frac{5 \pi}{12} \kappa_{i}+\frac{5 \pi}{16} \kappa_{i}^{2}\right) G_{i i}, \\
B_{i}\left(\rho_{i}^{*}\right) & =\frac{\sqrt{30}}{21} \rho_{i}^{*} \\
C_{i}\left(\rho_{i}^{*}, \kappa_{i}\right) & =\frac{19}{28} \rho_{i}^{*}-\frac{1}{18} \rho_{i}^{* 2}\left(\frac{9 \pi^{2}}{512}+\frac{\pi}{40} \kappa_{i}+\frac{9 \pi}{128} \kappa_{i}^{2}\right) G_{i i} \\
A_{A B}\left(\left\{\rho_{i}^{*}\right\},\left\{\kappa_{i}\right\}\right) & =\frac{1}{5} \rho_{A}^{*} \rho_{B}^{*}\left(\frac{5 \pi^{2}}{64}-\frac{5 \pi}{24}\left(\kappa_{A}+\kappa_{B}\right)+\frac{5 \pi}{32} \kappa_{A} \kappa_{B}\right) G_{A B} \\
C_{A B}\left(\left\{\rho_{i}^{*}\right\},\left\{\kappa_{i}\right\}\right) & =\frac{1}{9} \rho_{A}^{*} \rho_{B}^{*}\left(\frac{9 \pi^{2}}{512}+\frac{\pi}{80}\left(\kappa_{A}+\kappa_{B}\right)+\frac{9 \pi}{256} \kappa_{A} \kappa_{B}\right) G_{A B} .
\end{aligned}
$$

An explicit expression for the quantities $G_{i j}$ in equations (20)-(23) results from a generalization of the Carnahan-Starling theory for HS systems to mixtures [54, 55, 56], yielding [43]

$$
G_{i j}=\frac{4-3\left(\rho_{i}^{*} \nu_{i}+\rho_{j}^{*} \nu_{j}\right)}{4\left(1-\rho_{i}^{*} \nu_{i}-\rho_{j}^{*} \nu_{j}\right)^{2}},
$$

where $\nu_{i}=v_{i} / d^{3}$.

\subsection{Equilibrium transition}

As a background for our later discussion of mixtures under shear we first investigate the isotropic-nematic transition in equilibrium. Specifically, we are interested in the stability of the two phases in dependence of the densities and aspect ratios. In equilibrium one expects a uniaxial nematic phase described by a single director, $\mathbf{n}$, characterizing the preferred alignment of the rods [57, 58]. The second rank tensor then reduces to $\mathbf{Q}^{i}=\sqrt{\frac{3}{2}} q_{i}\langle\overrightarrow{\boldsymbol{n n}}\rangle$, where $q_{i}$ is the eigenvalue related to $\boldsymbol{n}$. For the products appearing in (20) it follows that $\operatorname{Tr}\left(\mathbf{Q}^{i} \cdot \mathbf{Q}^{j}\right)=q_{i} q_{j}$ and $\operatorname{Tr}\left(\mathbf{Q}^{i} \cdot \mathbf{Q}^{i} \cdot \mathbf{Q}^{i}\right)=q_{i}^{3} / \sqrt{6}$. By definition of the second rank tensor [see (6) with $k=2$ ] the eigenvalues $q_{i}$ are related to the well known Maier-Saupe order parameter $S_{2}$ via $q_{i}=\sqrt{5} S_{2}$ [31].

As a further simplification, we focus on the so-called "semi-dilute" regime corresponding to low densities. Physically, this implies that the particles have few contacts [59] and that long-range hydrodynamic interactions are negligible [60]. On a more formal level, the low-density limit implies that the contact values of the pair correlation functions tend to one, and the same holds for their density averages. Thus, $G_{i j} \rightarrow 1$. Still, as we will demonstrate below for the case of a one-component system, the mesoscopic theory provides reliable results (in comparison to particle-based simulations [11]) in the range $\kappa=20-40$. We also note that although the choice $G_{i j}=1$ is the same as that in original Onsager theory [7], the present free energy functional is different due to the more sophisticated treatment of the excluded volume (see Appendix A). 
2.4.1. Single component system To validate our approach against literature results, we first consider the case of a one-component system. The orientational free energy then reduces to the well-known Landau free energy expression [30]

$$
\mathscr{F}^{\text {or }}=A\left(\rho^{*}, \kappa\right) q^{2}-\frac{B\left(\rho^{*}\right)}{\sqrt{6}} q^{3}+C\left(\rho^{*}, \kappa\right) q^{4},
$$

where the coefficients now depend on the aspect ratio and the density. From (27), we find the three stationary solutions (determined by $d \mathscr{F}^{\text {or }} / d q=0$ )

$$
\begin{aligned}
q^{0} & =0 \\
q^{ \pm} & =\frac{\sqrt{6} B}{16 C}\left(1 \pm \sqrt{1-\frac{64 A C}{3 B^{2}}}\right) .
\end{aligned}
$$

As usual, the isotropic phase becomes globally unstable (i.e., $d^{2} \mathscr{F}^{\text {or }} /\left.d q^{2}\right|_{q^{0}}<0$ ) when the second-order coefficient $A\left(\rho^{*}, \kappa\right)$ changes sign from positive and negative. In terms of the density, this implies that within the stable or metastable isotropic phase,

$$
\rho^{*}<5\left(\frac{5 \pi^{2}}{64}-\frac{5 \pi}{12} \kappa+\frac{5 \pi}{16} \kappa^{2}\right)^{-1} .
$$

Directly at $A=0$, the nematic state already exists as a metastable state. The corresponding value of the order parameter follows from (29) as $q^{c}=\sqrt{6} B / 8 C$. Further, from the second derivative evaluated at $q^{+}$one finds that the nematic state becomes globally unstable $\left(d^{2} \mathscr{F}^{\text {or }} /\left.d q^{2}\right|_{q^{ \pm}}<0\right)$ when $A>3 B^{2} / 64 C$. The free energies $\mathscr{F}^{\text {or }}\left(q^{0}\right)$ and $\mathscr{F}^{\text {or }}\left(q^{+}\right)$become equal at

$$
A_{n i}=\frac{B^{2}}{24 C}
$$

The resulting order parameter at isotropic-nematic coexistence is given by

$$
q_{0}=\frac{\sqrt{6} B}{12 C}
$$

The stability ranges following from the above analysis are illustrated in the state diagram in figure 1, where the axes are given by the density and the aspect ratio. Specifically, the (red) line in figure 1 represents the boundary of (meta-) stability of the isotropic state, determined by the maximal density fulfilling the inequality (30), as function of $\kappa$. On the other hand, the (black) $\boldsymbol{\nabla}$ line indicates the solutions of the relation $A=3 B^{2} / 64 C$ for a given value of $\kappa$. This line can be seen as the upper boundary of the stable isotropic state.

For very large aspect ratios, $\kappa>80$, our approach is consistent with Onsager's second virial theory [7]. For smaller aspect ratios, we compare our stability limits with the coexistence densities of isotropic and nematic phases (plotted in (blue) $\bullet$ and $\mathbf{\Delta}$ ) obtained by Bolhuis et al in [11] using Gibbs-ensemble simulations. We find that two sets of data agree reasonably well. Note that the orientational free energy (27) can, by definition, not predict the coexistence densities, since we have neglected the densities $\rho_{i}$ as order parameters. 


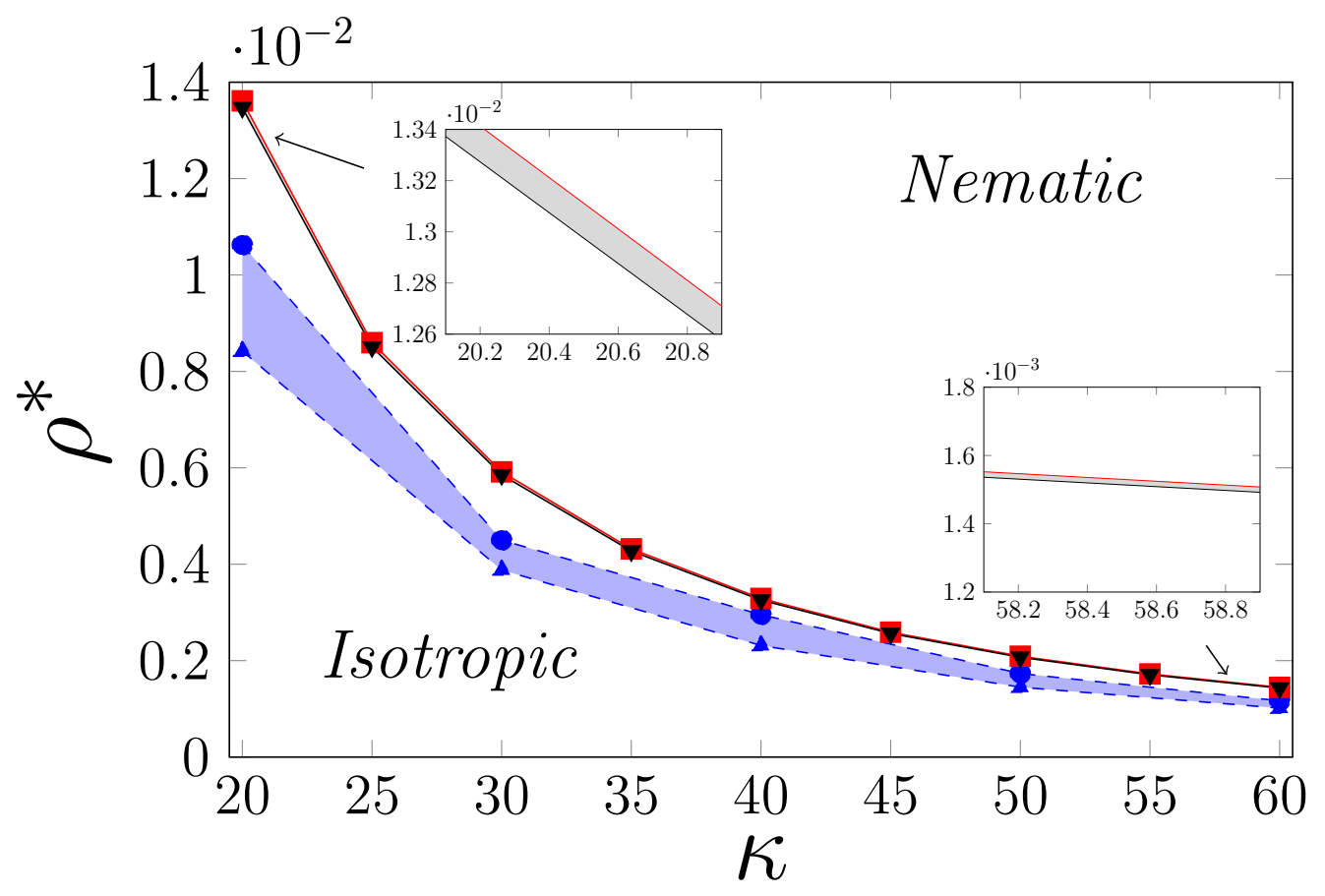

Figure 1. (Color online) Isotropic-nematic transition of a system of hard spherocylinders with aspect ratios between 20 and 60 . The (red) squares correspond to the lower limit of the density $\rho^{*}$ given by inequality (30), while (black) down-triangles are the numerical solutions of the relation $A=3 B^{2} / 64 C$. For comparison, the (blue) circles and up-triangles correspond to data for the isotropic-nematic phase coexistence from simulations of Bolhuis et al, see [1].

2.4.2. Binary mixture Depending on the overall density and concentrations, binary mixtures of particles with different aspect ratios can exhibit a very rich phase behavior [2, 61. Again, to calculate full phase diagrams, including first-order coexistence regions in density space, one would have to consider the full free energy given in equations (2) -(5) . Here we restricted ourselves to computing orientational state diagrams of the binary mixture by minimizing the orientational part of the free energy, $\mathscr{F}^{\text {or }}$, with respect to the uniaxial order parameters $q_{A}$ and $q_{B}$. The state diagrams are evaluated in the plane spanned by the total density $\rho^{*}=N d^{3} / V$ and concentration $x_{i}=N_{i} / N$ (such that $\rho_{i}^{*}=N d^{3} x_{i} / V=\rho^{*} x_{i}$ is the density of species $i$ ). We focus on two mixtures characterized by aspect ratios also occurring in real systems [2].

The methodology is the following. Using a standard Newton-Raphson minimization algorithm, we search for values of $q_{A}$ and $q_{B}$ corresponding to minima of $\mathscr{F}^{\text {or }}$. We then evaluate the orientational free energy at these points and determine the minimum value, $\min \left\{\mathscr{F}^{\text {or }}\right\}$. In the case that $\min \left\{\mathscr{F}^{\text {or }}\right\}=0$ the isotropic phase is stable whereas if $\min \left\{\mathscr{F}^{o r}\right\} \neq 0$ a nematic state exists. The result of this procedure is shown in figures $2(\mathrm{a}-\mathrm{b})$.

In both diagrams, figures 2(a) and 2(b), we see that for relatively low densities, $\rho^{*} \sim 0.005$, the isotropic state is stable for all concentrations. As the total density of the system increases, the isotropic phase becomes unstable with respect to the nematic, and 


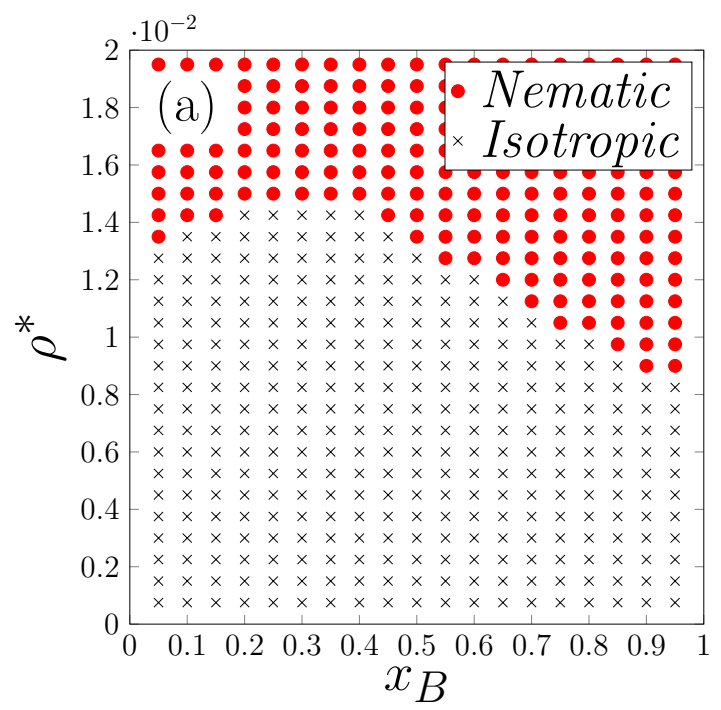

(a) $\kappa_{A}=20, \quad \kappa_{B}=25$.

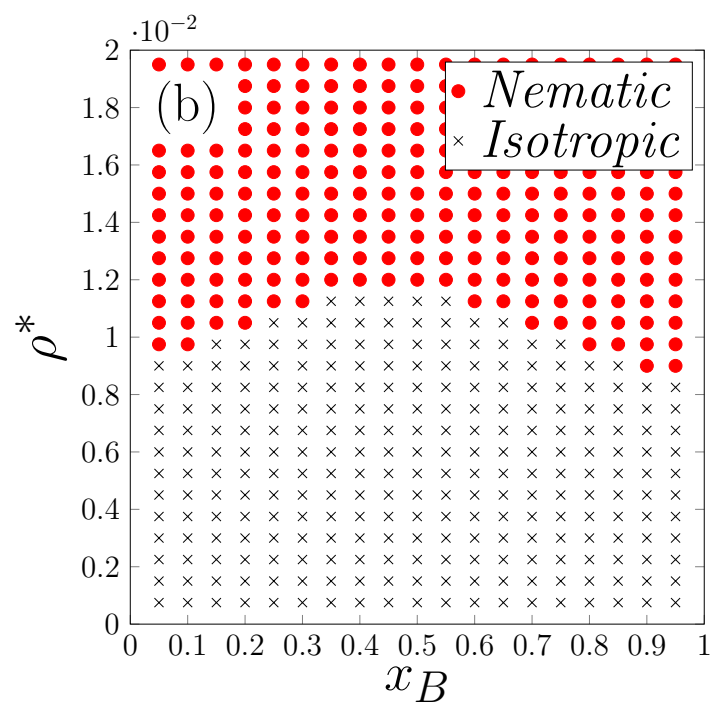

(b) $\kappa_{A}=24, \quad \kappa_{B}=25$.

Figure 2. State diagrams for a mixture of long and short hard spherocylinders with uniaxial ordering. The nematic ordering is marked with $(\bullet)$, whereas $(+)$ denotes an isotropic state. In the limit where $x_{B}=0$ these diagrams coincide with the one component results portrayed in figure 1

for sufficiently high densities, $\rho^{*} \sim 0.020$, the system is in the nematic state irrespective of the concentration.

As expected, the mole fraction of long spherocylinders plays an important role for the location of the transition. This is observed by focusing on a constant density value, $\rho^{*} \sim 0.0075$ in figure 2(b). For $x_{B}=0.2$ the nematic phase does not exist, but as $x_{B}$ increases, $x_{B}=0.8$, the nematic state appears. In the limiting case where $x_{B}=0$ the results coincide with the one component system presented in figure 1 .

Clearly, it would be very interesting to compare the equilibrium behavior found here to that of real systems. However, to our knowledge, experimental results have only be reported on binary mixtures of fd-viruses with equal length and different diameters [2]. This is different from the mixture of rods with different lengths and equal diameters considered in figure 2. In principle, one could apply the present theory also to the first case by rescaling the number densities via the length (instead of the diameter) of the particles and recalculating the excluded volume coefficients in equations (21)(25). Here we concentrate on the case of different length, the final motivation being the non-equilibrium behavior of rod-disk mixtures such as (models of) blood [15].

2.4.3. Free energy scaling For the subsequent analysis it is convenient to re-write (20) into a dimensionless form using an appropriate scaling of the free energy. In principle, different scalings are possible. Here we follow the strategy used in earlier studies [26, 27, 29, 31] of one-component systems.

Specifically, we introduce the scaled alignment tensors, $\tilde{\mathbf{Q}}^{i}$ and the scaled free 
energy, $\mathscr{F}_{\text {ref }}^{\text {or }}$, as

$$
\tilde{\mathrm{Q}}^{i}=\frac{\mathrm{Q}^{i}}{q_{0}^{i}} \quad \text { and } \quad \tilde{\mathscr{F}}^{\text {or }}=\frac{\mathscr{F}^{\text {or }}}{\mathscr{F}_{\text {ref }}^{\text {or }}}
$$

where $q_{0}^{i}$ is the coexistence value of the uniaxial order parameter of the corresponding one-component system given in (32), and $\mathscr{F}_{r e f}^{o r}=2 C_{A B}\left(q_{0}^{A} q_{0}^{B}\right)^{2}$. In the latter expression, $C_{A B}$ is the coupling coefficient of the fourth-order term $\left(\mathbf{Q}^{A}: \mathbf{Q}^{B}\right)^{2}$ of the free energy (20).

To simplify the expression for the scaled free energy resulting from inserting (33) into (20), we make the following assumptions. First, we set the coefficients $B_{i}$ and $C_{i}$ [see equations (23) and (24), respectively] equal to their (positive) values at I-N coexistence of the one-component system, that is,

$$
B_{i}=B_{i}\left(\rho_{i_{n i}}^{*}\right) \quad \text { and } \quad C_{i}=C_{i}\left(\rho_{i_{n i}}^{*}, \kappa_{i}\right),
$$

where the densities $\rho_{i_{n i}}^{*}$ are calculated by solving (31). Thus, we henceforth neglect changes of $B_{i}$ and $C_{i}$ with $\rho_{i}$. We further suppose that the fourth-order coefficient of the one-component system, $C_{A}$ and $C_{B}$, are related to $C_{A B}$ via

$$
C_{A B}=C_{A}\left(\frac{q_{0}^{A}}{q_{0}^{B}}\right)^{2}=C_{B}\left(\frac{q_{0}^{B}}{q_{0}^{A}}\right)^{2} .
$$

Equation (35) implies that for given values of $\kappa_{i}$ and $\rho_{i}^{*},\left|C_{A B}^{2}-C_{A} C_{B}\right|=0$. To test this assumption for a representative example, we consider a binary mixture in the nematic state $\left(\rho^{*}=0.002\right)$ where $30 \%$ of the particles have an aspect ratio $\kappa_{A}=20$ and the others have an aspect ratio $\kappa_{B}=25$. In this case we find $\left|C_{A B}^{2}-C_{A} C_{B}\right| \approx 3.79 \times 10^{-7}$, which makes our ansatz plausible.

With the simplifications (34)-(35), the scaled version of the orientational free energy [see (20)] becomes

$$
\begin{aligned}
\tilde{\mathscr{F}}^{o r}\left[\tilde{\mathbf{Q}}^{A}, \tilde{\mathbf{Q}}^{B}\right]= & \frac{\mathscr{F}^{o r}\left[\mathbf{Q}^{A}, \mathbf{Q}^{B}\right]}{\mathscr{F}_{r e f}^{o r}} \\
= & \sum_{i=A}^{B}\left\{\frac{\Theta_{i}}{2}\left(\tilde{\mathbf{Q}}^{i}: \tilde{\mathbf{Q}}^{i}\right)-\sqrt{6} \operatorname{Tr}\left(\tilde{\mathbf{Q}}^{i} \cdot \tilde{\mathbf{Q}}^{i} \cdot \tilde{\mathbf{Q}}^{i}\right)+\frac{1}{2}\left(\tilde{\mathbf{Q}}^{i}: \tilde{\mathbf{Q}}^{i}\right)^{2}\right\} \\
& -\frac{\Theta_{A B}}{2}\left(\tilde{\mathbf{Q}}^{A}: \tilde{\mathbf{Q}}^{B}\right)-\frac{1}{2}\left(\tilde{\mathbf{Q}}^{A}: \tilde{\mathbf{Q}}^{B}\right)^{2} .
\end{aligned}
$$

In (36), the coefficients $\Theta_{i}$ of the second-orders term are given by

$$
\Theta_{i}=24 \frac{A_{i}\left(\rho_{i}^{*}, \kappa_{i}\right) C_{i}\left(\rho_{i_{n i}}^{*}, \kappa_{i}\right)}{B_{i}\left(\rho_{i_{n i}}^{*}\right)^{2}}=\frac{A_{i}\left(\rho_{i}^{*}, \kappa_{i}\right)}{A_{n i}\left(\rho_{i_{n i}}^{*}, \kappa_{i}\right)},
$$

which shows the explicit dependence of $\Theta_{i}$ on the composition (that is, on $\rho_{i}$ ) and $\kappa$. The remaining coefficient $\Theta_{A B}$ is a positive quantity which is depends not on the composition, but only on the aspect ratios. Explicitly,

$$
\Theta_{A B}=\frac{9\left[\frac{5 \pi^{2}}{64}-\frac{5 \pi}{24}\left(\kappa_{A}+\kappa_{B}\right)+\frac{5 \pi}{32} \kappa_{A} \kappa_{B}\right]}{5\left[\frac{9 \pi^{2}}{512}+\frac{\pi}{80}\left(\kappa_{A}+\kappa_{B}\right)+\frac{9 \pi}{256} \kappa_{A} \kappa_{B}\right] q_{0}^{A} q_{0}^{B}} .
$$




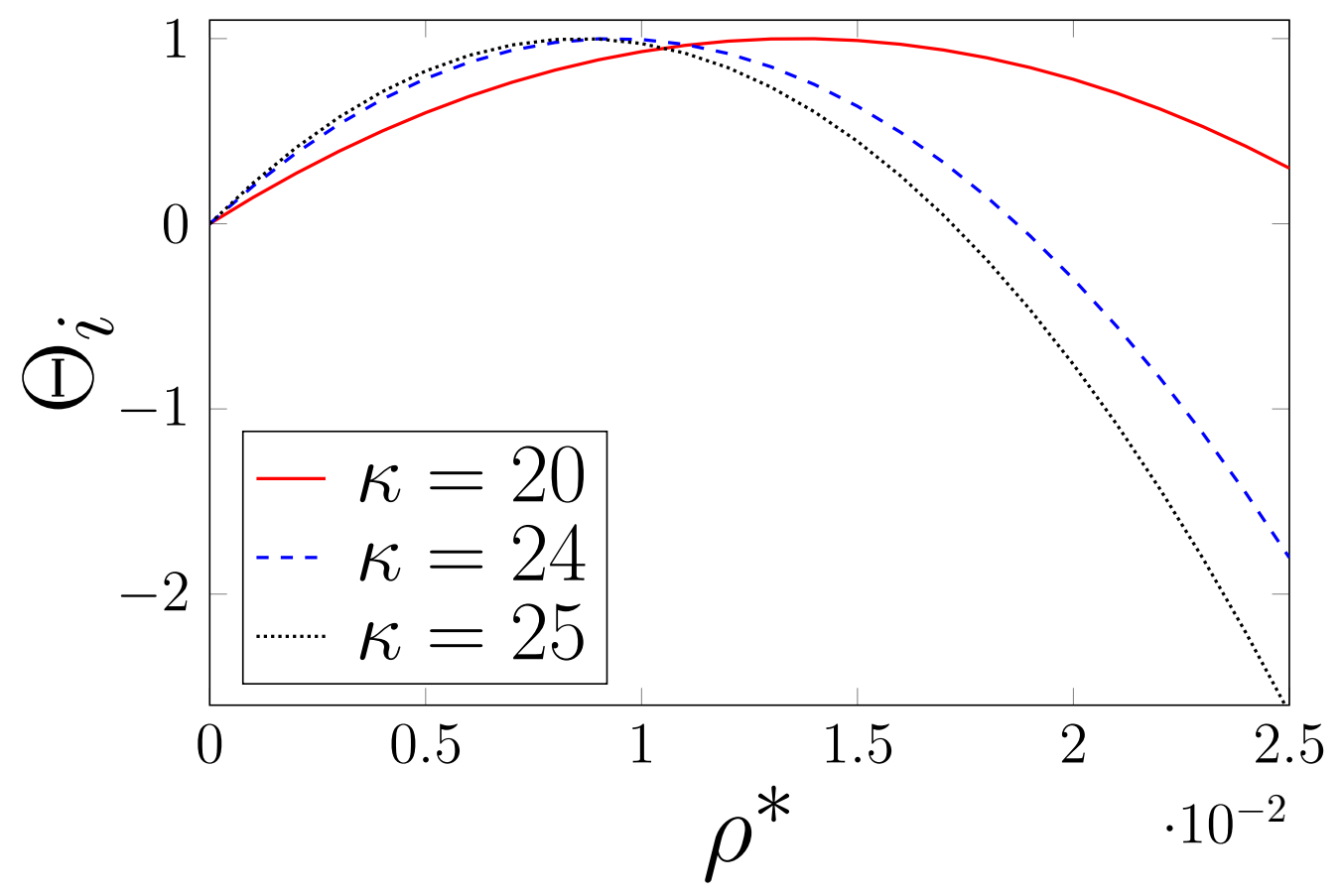

Figure 3. Relation of $\Theta_{i}$ versus the reduced density, given by (37), for equimolar binary mixtures $\left(x_{A}=x_{B}=0.5\right)$ and different aspect ratio. The coexistence densities $\rho_{i_{n i}}^{*}$ are given by the roots of (31).

In the limiting case of the one-component system, the scaled free energy (36) reduces to the corresponding expression given in [26, 27, 29, 31].

2.4.4. Analysis of the scaled free energy To better understand the properties of the scaled free energy we consider, first, the explicit density dependence of the coefficients $\Theta_{i}$ appearing in front of the quadratic powers of $\tilde{\mathbf{Q}}^{i}$ [see (37)]. As an illustration, we show in figure 3 numerical results for an equimolar binary mixture and three values of the aspect ratio (note that each $\Theta_{i}$ depends only on $\kappa_{i}$ ). One observes the same qualitative behavior irrespective of the actual value of $\kappa$ : the coefficient $\Theta_{i}$ first increases with density and then reaches a maximum, after which it monotonically decreases and changes sign from positive to negative. This change of sign is expected (within the Landau picture) for a system displaying a phase transition. The corresponding density is the smaller, the larger $\kappa$.

To examine the relation between the sign of $\Theta_{i}$ and the mixture's stability, we consider the case of uniaxial order. In this case (36) reduces to

$$
\tilde{\mathscr{F}}_{\text {uni }}^{\text {or }}=\sum_{i=A}^{B}\left\{\frac{\Theta_{i}}{2} \tilde{q}_{i}^{2}-\tilde{q}_{i}^{3}+\frac{1}{2} \tilde{q}_{i}^{4}\right\}-\frac{\Theta_{A B}}{2} \tilde{q}_{A} \tilde{q}_{B}-\frac{1}{2} \tilde{q}_{A}^{2} \tilde{q}_{B}^{2},
$$



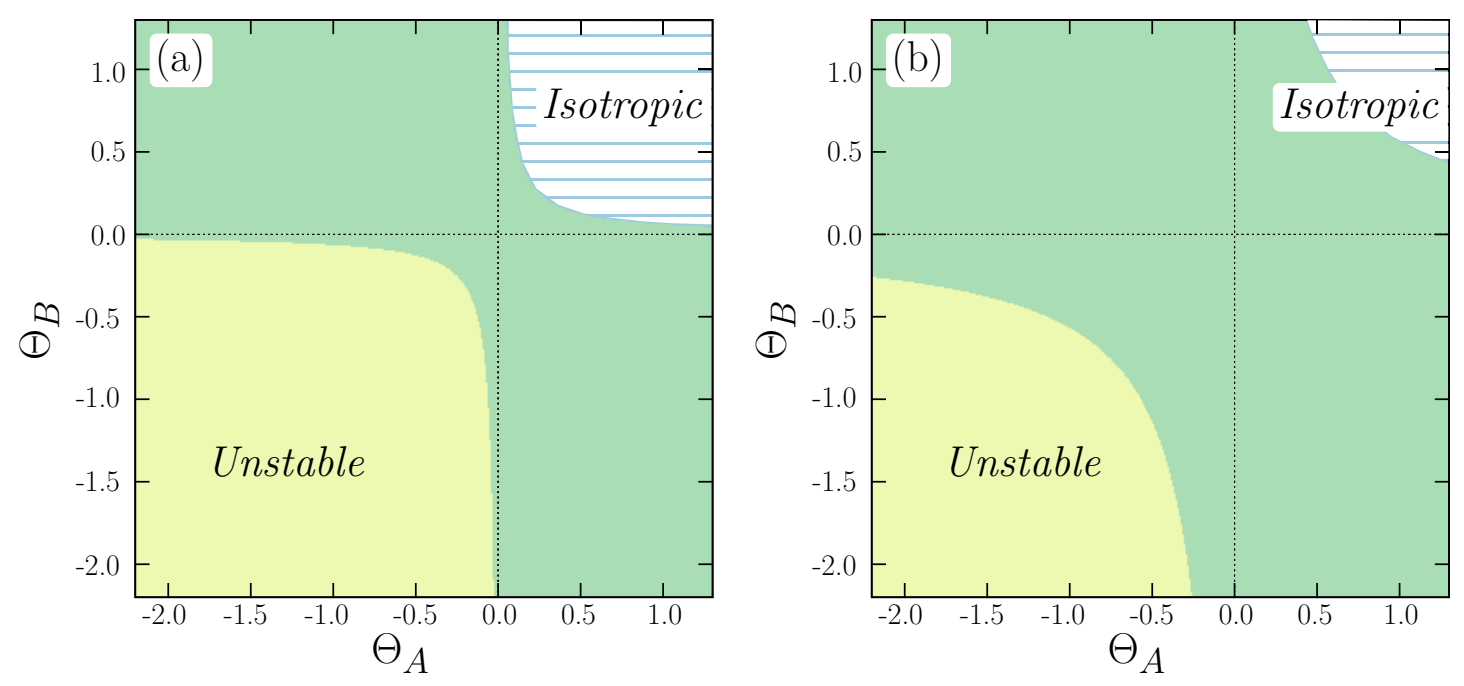

Figure 4. Stability regions of the isotropic state $\left(\tilde{\mathbf{q}}_{t}=(0,0)\right)$ according to (41). The values of $\Theta_{A B}$ are (a) $\Theta_{A B}=0.50$ and (b) $\Theta_{A B}=1.50$.

where $\tilde{q}_{i}=q_{i} / q_{0}^{i}$. The stability of a given minimum $\tilde{\mathbf{q}}$ can be determined from the Hessian matrix [62]

$$
H\left(\tilde{q}_{A}, \tilde{q}_{B}\right)=\left(\begin{array}{cc}
\Theta_{A}-6 \tilde{q}_{A}+6 \tilde{q}_{A}^{2}-\tilde{q}_{B}^{2} & -\frac{\Theta_{A B}}{2}-2 \tilde{q}_{A} \tilde{q}_{B} \\
-\frac{\Theta_{A B}}{2}-2 \tilde{q}_{A} \tilde{q}_{B} & \Theta_{B}-6 \tilde{q}_{B}+6 \tilde{q}_{B}^{2}-\tilde{q}_{A}^{2}
\end{array}\right)
$$

evaluated of the minimum. We now focus on the stability of the isotropic state, $\tilde{\mathbf{q}}_{t}=(0,0)$. Stability then requires that the two eigenvalues or, alternatively, the two diagonal elements $\Theta_{i}$ and the determinant

$$
\operatorname{det}\left[H\left(\tilde{\mathbf{q}}_{t}\right)\right]=\Theta_{A} \Theta_{B}-\frac{\Theta_{A B}^{2}}{4}>0,
$$

are positive (where we recall that $\Theta_{A B}$ is a positive quantity). An illustration of the regions of stability of the isotropic phase in the $\Theta_{A}-\Theta_{B}$ plane at fixed $\Theta_{A B}$ is given in figure 4. The larger $\Theta_{A B}$, the smaller is the region where the isotropic state is a minimum of the free energy.

It seems straightforward to repeat the same analysis for free energy minima corresponding to nematic states $\left(q_{A} \neq 0, q_{B} \neq 0\right)$. However, in that context we face a problem already discussed in the context of one-component systems [36, 63], which concerns the bounds of possible values. This can be seen as follows: The order parameters $q_{A}$ and $q_{B}$ are related to the Maier-Saupe order parameter $S_{2}$ by $q_{i}=\sqrt{5} S_{2}$ [31]. The latter lies within the range $-1 / 2 \leq S_{2} \leq 1$. The bounds of the corresponding scaled order parameters, $q_{A}$ and $q_{B}$, involve the factor $1 / q_{0}^{i}$. Using (32) and the values of the coexisting densities $\rho_{i_{n i}}^{*}$ given by the roots of (31), we find that the scaled order parameters are defined in the range

$$
-1.30 \lesssim q_{i} \lesssim 2.60
$$


Table 1. Numerical values of the constants $\Theta_{A}, \Theta_{B}$ and $\Theta_{A B}$ for equimolar binary mixtures $\left(x_{A}=x_{B}=0.5\right)$. This values correspond to different points in the phase diagrams in figures $3(\mathrm{a}-\mathrm{b})$ and are calculated using (37) and (38).

\begin{tabular}{ccccccc}
\hline & \multicolumn{2}{c}{ Figure 2(a) } & \multicolumn{2}{c}{ Figure 2(b) } & \multicolumn{2}{c}{ Equation (41) } \\
$\rho^{*}$ & \multicolumn{2}{c}{$\Theta_{i}$} & \multicolumn{2}{c}{$\Theta_{i}$} & $\operatorname{det}\left[H\left(\tilde{\mathbf{q}}_{t}\right)\right]$ & \\
$\left(10^{-2}\right)$ & $\kappa_{A}=20$ & $\kappa_{B}=25$ & $\kappa_{A}=24$ & $\kappa_{B}=25$ & & \\
\hline 0.50 & 0.599 & 0.825 & 0.783 & 0.825 & + & Isotropic \\
1.00 & 0.929 & 0.973 & 0.995 & 0.973 & + & Isotropic \\
1.75 & 0.918 & -0.073 & 0.239 & -0.073 & - & Unstable \\
2.00 & 0.779 & -0.759 & -0.298 & -0.759 & - & Unstable \\
\hline \multicolumn{7}{c}{$\Theta_{A B}=1.223$} \\
\hline
\end{tabular}

However, free minimization of the scaled free energy (36) does not automatically respect these bounds; rather it yields, for a range of parameters, nematic minima outside the allowed domain. To solve this problem, one could consider an "amended" version of the free energy, similar to what has been done for one-component systems [36, 63]. However, this would go beyond the scope of the present work.

Another question, given the simplifications involved in our scaling of the free energy, is to which extent the stability conditions for the isotropic phase found here are consistent with our earlier results obtained by minimization of the unscaled free energy (see figure 2). As a check we have evaluated the quantities $\Theta_{i}$ for several state points in the diagrams plotted in figure 2 The results are summarized in table1, showing the consistency of unscaled and scaled free energy.

\section{Shearing within the nematic state}

Based on the mesoscopic free energy functional derived in the preceding section we now explore the dynamical behavior of the system under shear. To this end we use a straightforward extension of the Doi-Hess equations [26, 27, 28], which may be derived either from the Fokker-Planck equation for the orientational distribution function or via irreversible thermodynamics (for a recent formulation of the derivation, see [31]). The extension for binary mixtures involves two coupled equations for the order parameter tensors $\mathbf{Q}^{A}$ and $\mathbf{Q}^{B}$ in presence of a flow field $\mathbf{v}$. Specializing on spatially homogeneous states the equations read

$$
\begin{aligned}
& \frac{d \mathbf{Q}^{A}}{d t}=2 \overparen{\Omega \cdot \mathbf{Q}^{A}}+2 \sigma \overparen{\Gamma \cdot \mathbf{Q}^{A}}-\frac{\Phi_{\mathbf{A}}^{\prime}}{\tau_{A}}-\sqrt{2} \frac{\tau_{A p}}{\tau_{A}} \boldsymbol{\Gamma}, \\
& \frac{d \mathbf{Q}^{B}}{d t}=2 \overparen{\Omega \cdot \mathbf{Q}^{B}}+2 \sigma \overparen{\Gamma \cdot \mathbf{Q}^{B}}-\frac{\Phi_{\mathbf{B}}^{\prime}}{\tau_{B}}-\sqrt{2} \frac{\tau_{B p}}{\tau_{B}} \boldsymbol{\Gamma},
\end{aligned}
$$


where $\boldsymbol{\Omega}=(1 / 2)\left(\nabla \mathbf{v}^{T}-\nabla \mathbf{v}\right)$ and $\boldsymbol{\Gamma}=(1 / 2)\left(\nabla \mathbf{v}^{T}+\nabla \mathbf{v}\right)$ are tensors describing the flow vorticity and flow deformation, respectively, and $\boldsymbol{\Phi}_{i}^{\prime}$ are derivatives of the (unscaled) orientational free energy, i.e.

$$
\boldsymbol{\Phi}_{i}^{\prime}=\frac{\partial \mathscr{F}^{o r}\left(\mathbf{Q}^{A}, \mathbf{Q}^{B}\right)}{\partial \mathbf{Q}^{i}} .
$$

The degree of the perturbation exerted by the shear flow is measured through the prefactor of the last term of equations (43)-(44), that is, the $\tau_{i p} / \tau_{i}$, which defines the so-called tumbling parameter via $\lambda_{i}=-\tau_{i p} / \tau_{i}$. Both $\tau_{i}$ and $\tau_{i p}$ are phenomenological relaxation times dependent on the solvent's viscosity and the effective gyration radius of the colloidal particles [27]. It can be shown that their ratio, and thus, $\lambda_{i}$, is related to the particle aspect ratio [27] via

$$
\lambda_{i}=-\frac{\tau_{i p}}{\tau_{i}}=\sqrt{\frac{3}{5}} \frac{\kappa_{i}^{2}-1}{\kappa_{i}^{2}+1} .
$$

For spherical particles $\lambda=0$, whereas for prolate and oblate particles, $\lambda>0$ and $\lambda<0$, respectively.

\subsection{Scaled dynamical equations}

In the following we specialize to a planar Couette shear flow characterized by the velocity $\mathbf{v}=\dot{\gamma} y \hat{e}_{x}$, where $\dot{\gamma}$ is the shear rate. Further, we re-introduce the scaled alignment tensors $\tilde{\mathbf{Q}}^{i}$ and the scaled free energy according to (33). Equations (43) and (44) then become

$$
\begin{aligned}
& \frac{d \tilde{\mathbf{Q}}^{A}}{d \tilde{t}}=2 \tilde{\dot{\gamma}} \tilde{\mathbf{\Omega}} \cdot \tilde{\mathbf{Q}}^{A}+2 \sigma \tilde{\dot{\gamma}} \tilde{\boldsymbol{\Gamma}} \cdot \tilde{\mathbf{Q}}^{A}-\tilde{\Phi_{A}^{\prime}}+\sqrt{2} \tilde{\lambda_{A}} \tilde{\dot{\gamma}} \tilde{\boldsymbol{\Gamma}}, \\
& \frac{d \tilde{\mathbf{Q}}^{B}}{d \tilde{t}}=2 \tilde{\dot{\gamma}} \tilde{\boldsymbol{\Omega}} \cdot \tilde{\mathbf{Q}}^{B}+2 \sigma \tilde{\dot{\gamma}} \tilde{\tilde{\boldsymbol{\Gamma}} \cdot \tilde{\mathbf{Q}}^{B}}-\tilde{\Phi_{B}^{\prime}}+\sqrt{2} \tilde{\lambda_{B}} \tilde{\dot{\gamma}} \tilde{\boldsymbol{\Gamma}},
\end{aligned}
$$

where $\tilde{t}=t / t_{\text {ref }}$ and $\tilde{\dot{\gamma}}=\dot{\gamma} t_{\text {ref }}$ are the re-scaled time and shear rate, and the reference time connected to the phenomenological relaxation times $\tau_{i}$, is $t_{\text {ref }}=\tau_{i}\left(q_{0}^{i}\right)^{2} \mathscr{F}_{\text {ref }}^{\text {or }}{ }^{-1}$.

The scaled vorticity and deformation tensors are $\tilde{\boldsymbol{\Omega}}=(1 / 2)\left(\hat{e}^{x} \hat{e}^{y}-\hat{e}^{y} \hat{e}^{x}\right)$ and $\tilde{\boldsymbol{\Gamma}}=$ $(1 / 2)\left(\hat{e}^{x} \hat{e}^{y}+\hat{e}^{y} \hat{e}^{x}\right)$, respectively. Further, the scaled tumbling parameters are given by

$$
\tilde{\lambda}_{i}=\lambda_{i} / q_{0}^{i}=\sqrt{\frac{3}{5}} \frac{1}{q_{0}^{i}} \frac{\kappa_{i}^{2}-1}{\kappa_{i}^{2}+1} .
$$

To give an example of typical values of the scaled tumbling parameters, we consider systems involving the PEG-coated $f d$-virus, like the ones used in the experiments of thick and thin colloidal rods of Purdy et al. [2]. For these systems, the Maier-Saupe order parameter at coexistence is $S_{0}=\left\langle P_{2}\right\rangle \approx 0.5--0.8$ [64]. Using $q_{0}^{i}=\sqrt{5} S_{0}$ [31] one obtains $\tilde{\lambda}_{i} \sim 0.43--0.69$. Finally, the derivative of the scaled free energy [see (36)] is given by

$$
\Phi_{i}^{\prime}=\Theta_{i} \tilde{\mathbf{Q}}^{i}-2 \sqrt{6} \overline{\tilde{\mathbf{Q}}^{i} \cdot \tilde{\mathbf{Q}}^{i}}+2\left(\tilde{\mathbf{Q}}^{i}: \tilde{\mathbf{Q}}^{i}\right) \cdot \tilde{\mathbf{Q}}^{i}-\frac{1}{2} \Theta_{A B} \tilde{\mathbf{Q}}^{j}-\left(\tilde{\mathbf{Q}}^{A}: \tilde{\mathbf{Q}}^{B}\right) \cdot \tilde{\mathbf{Q}}^{j}
$$




\subsection{Explicit equations of motion}

For a practical analysis of equations (47) and (48), the second-order tensors $\tilde{\mathbf{Q}}^{i}$ are expanded in terms of a standard tensorial basis with five independent elements, see [63, 35, 31]. Introducing this tensor basis results into a system of ten coupled nonlinear equations for $q_{0}^{i}, q_{1}^{i}, \cdots, q_{4}^{i}, i=A, B$. These equations are given by

$$
\begin{aligned}
& \dot{q}_{0}^{i}=-\left(\phi_{0}^{i}+\varphi_{0}^{j}\right)-\frac{\sqrt{3}}{3} \dot{\gamma} \sigma q_{2}^{i}, \\
& \dot{q}_{1}^{i}=-\left(\phi_{1}^{i}+\varphi_{1}^{j}\right)+\dot{\gamma} q_{2}^{i}, \\
& \dot{q}_{2}^{i}=-\left(\phi_{2}^{i}+\varphi_{2}^{j}\right)-\dot{\gamma} q_{1}^{i}-\frac{\sqrt{3}}{3} \dot{\gamma} \sigma q_{0}^{i}+\lambda_{i} \dot{\gamma}, \\
& \dot{q}_{3}^{i}=-\left(\phi_{3}^{i}+\varphi_{3}^{j}\right)+\frac{1}{2} \dot{\gamma}(\sigma+1) q_{4}^{i}, \\
& \dot{q}_{4}^{i}=-\left(\phi_{4}^{i}+\varphi_{4}^{j}\right)+\frac{1}{2} \dot{\gamma}(\sigma-1) q_{3}^{i} .
\end{aligned}
$$

In these equations, the quantities $\phi_{k}^{i}$, which describe the relaxation of the particles towards equilibrium, are given by

$$
\begin{aligned}
& \phi_{0}^{i}=q_{0}^{i}\left(\Theta_{i}-3 q_{0}^{i}+2 \mathfrak{q}_{i}^{2}\right)+3\left(q_{1}^{i 2}+q_{2}^{i 2}\right)-\frac{3}{2}\left(q_{3}^{i 2}+q_{4}^{i 2}\right), \\
& \phi_{1}^{i}=q_{1}^{i}\left(\Theta_{i}+6 q_{0}^{i}+2 \mathfrak{q}_{i}^{2}\right)-\frac{3}{2} \sqrt{3}\left(q_{3}^{i 2}-q_{4}^{i 2}\right), \\
& \phi_{2}^{i}=q_{2}^{i}\left(\Theta_{i}+6 q_{0}^{i}+2 \mathfrak{q}_{i}^{2}\right)-3 \sqrt{3} q_{3}^{i} q_{4}^{i}, \\
& \phi_{3}^{i}=q_{3}^{i}\left(\Theta_{i}-3 q_{0}^{i}+2 \mathfrak{q}_{i}^{2}\right)-3 \sqrt{3}\left(q_{1}^{i} q_{3}^{i}+q_{2}^{i} q_{4}^{i}\right), \\
& \phi_{4}^{i}=q_{4}^{i}\left(\Theta_{i}-3 q_{0}^{i}+2 \mathfrak{q}_{i}^{2}\right)+3 \sqrt{3}\left(q_{1}^{i} q_{4}^{i}-q_{2}^{i} q_{3}^{i}\right) .
\end{aligned}
$$

while the quantities $\varphi_{k}^{i}$, which take into account the inter-component interaction within the mixture, are given by

$$
\varphi_{\alpha}^{j}=-q_{\alpha}^{j}\left(\Theta_{A B}+\mathfrak{q}_{A B}^{2}\right),
$$

where $\alpha=0,1, \cdots, 4$. In equations (52)-(53), $\mathfrak{q}_{i}^{2}=\sum q_{k}^{i 2}$ and $\mathfrak{q}_{A B}^{2}=\sum q_{k}^{i} q_{k}^{j}$.

For one-component systems, it can be shown by general arguments [34] (and has been demonstrated numerically [32, 33]) that the structure of equations (51)-(53) leads to various types of oscillatory solutions provided that the shear rate $\dot{\gamma} \neq 0$. In particular, for the case $\sigma=0$ (and appropriate thermodynamic state points, usually within the nematic state) the equations predict the presence of two oscillatory "subunits" indicating oscillations within the shear plane $\left(q_{1} \neq 0, q_{2} \neq 0, q_{3}=q_{4}=0\right)$ and out of the shear plane $\left(q_{3} \neq 0, q_{4} \neq 0\right)$, respectively. These oscillations have also been observed in numerous numerical studies [65, 66]. Here we follow these previous studies of onecomponent systems and set $\sigma=0$. Given the fact that our set of equations is just a direct extension of those for one-component systems, we expect a variety of oscillatory solutions also in the mixture case.

To perform the numerical integration of equations (51)-(53) we employ a standard Runge-Kutta algorithm with adaptive step size control [67]. The starting values of the 
tensorial components $q_{0}^{i}, \cdots, q_{4}^{i}$ (where $i=A, B$ ) are chosen randomly in the interval $[0,1]$, using a uniform distribution. We note that the choice of initial values and the overall number of time-steps required to obtain reliable results (disregarding transient initial behavior) strongly depends on the chosen parameters. Therefore, all numerical integrations have been repeated several times. To characterize the resulting dynamical states, we use an algorithm that recognizes the periodicity and sign change of the calculated time-dependent order parameters $q_{k}^{i}(t)$ [68, 32, 35, 65, 69, 34].

\subsection{Numerical results}

In the following we investigate shear-driven systems whose equilibrium state $(\dot{\gamma}=0)$ is nematic. Specifically, we set $\Theta_{A}=\Theta_{B}=-0.25$. According to the stability analysis in section 2.4.4, the isotropic phase is then unstable irrespective of $\Theta_{A B}$.

The section is divided into two parts. First, we discuss the effect of varying the shear rate, $\dot{\gamma}$, and the tumbling parameter of species $\mathrm{B}, \lambda_{B}$, while $\lambda_{A}$ is fixed. We note that in real experiments, variation of the tumbling parameter is somewhat difficult since each value of this parameter corresponds to a different aspect ratio (and thus, different particle type), see (49). However, in the framework of our dynamical equations, $\lambda_{B(A)}$ is the crucial parameter measuring the impact of the shear-induced perturbation, thus it seems worth to explore its role. Specifically, we set $\lambda_{A}=1.2=$ const and consider a range of values $\lambda_{B}>\lambda_{A}$, implying that the $\mathrm{B}$-particles have larger aspect ratios. The second part of the section then addresses the role of the "cross-coupling" parameter $\Theta_{A B}$.

3.3.1. Variation of the tumbling parameter and the shear rate The numerical integration procedure described in section 3.2 leads to dynamical state diagrams for each of the two species which we present in figure 5 .

Each colored region corresponds to a different dynamical state characterized by a specific behavior of the respective nematic director, that is, the eigenvector related to the largest eigenvalue of the ordering tensor $\mathbf{Q}^{A(B)}$. Specifically, we observe wagging states $(\mathrm{W})$, tumbling states (T), kayaking-wagging states (KW), kayaking-tumbling states (KT), and (flow-)alignment states (A). The last type of states corresponds to fixed points of the dynamical system, where the director is "frozen" along a direction within the shear plane. All other states mentioned so far are characterized by a time-dependent, regular oscillatory behavior of the coefficients $q_{i}(t)$ of $\mathbf{Q}(t)$. Physically, these oscillations correspond to oscillations of the nematic director either within the shear plane (W, $\mathrm{T}$ ), or out of the shear plane (KW, KT). The boundaries between the colored regions in figure 5 correspond to different types of dynamical bifurcations. The simplest one is a (supercritical) Hopf bifurcation occurring at the boundary between alignment (A) and wagging $(\mathrm{W})$. In [34] we have presented a complete continuation analysis revealing the complexity of the dynamical behavior already in the one-component case (where one has already five dynamical variables). Performing such an analysis for the mixture 

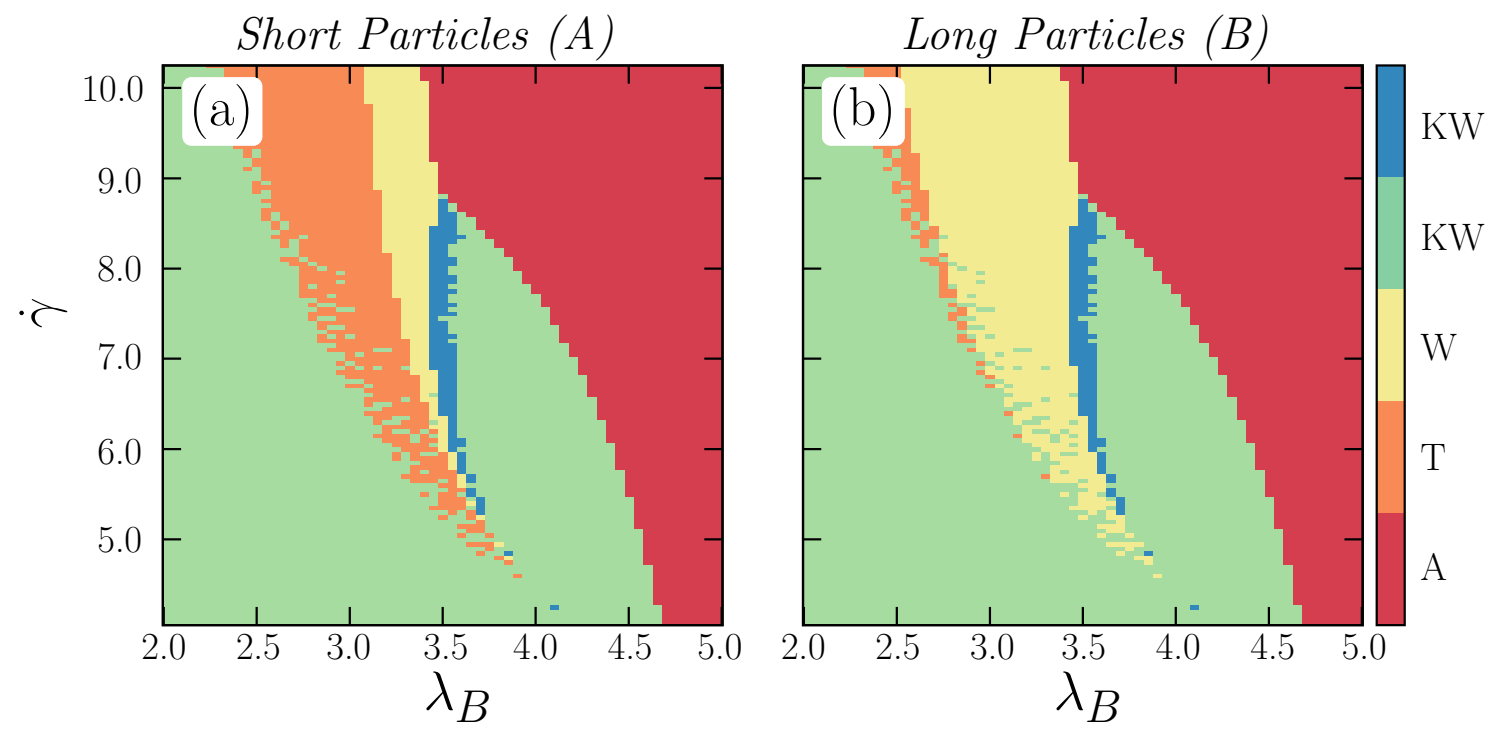

Figure 5. Dynamical state diagram for a binary mixtures characterized by $\Theta_{A}=$ $-0.25, \Theta_{B}=-0.25, \Theta_{A B}=1.50$, and $\lambda_{A}=1.2$. Parts a) and b) illustrate the behavior of the short and long particles, respectively. The areas are computed via direct numerical integration. Each colored region corresponds to different dynamical behavior (see color bar on the right side): $\mathrm{A}=$ Alignment, $\mathrm{W}=$ Wagging, $\mathrm{T}=$ Tumbling, $\mathrm{KW}$ = Kayak-Wagging, $\mathrm{KT}=$ Kayak-Tumbling.

would have been beyond the scope for the present paper. We therefore just note that the shape of each state diagram in figure 5 is qualitatively similar to that of one-component systems [32, 33] sheared from a nematic equilibrium state, apart from the fact that the latter also contain a chaotic region (C) [32, 35, 33, 70]. Here, we did not detect such a region because our algorithm does not calculate Lyapunov exponents. Still, we expect that such a region also occurs in the mixture system (for both species) at values of $\dot{\gamma}$, $\lambda_{B}$ where the regions $\mathrm{A}, \mathrm{KW}$ and $\mathrm{KT}$ meet.

While the overall behavior of the two species is clearly very similar, there are interesting differences for specific values of $\dot{\gamma}$ and $\lambda_{B}$. As an example, consider the shear rate $\dot{\gamma}=7.5$ and $\lambda_{B}=3.0$. At these parameters, the long rods are in the $\mathrm{W}$ state while the short rods are in a tumbling $(\mathrm{T})$ state. In other words, both directors display oscillatory behavior within the shear plane, but with different characteristics: Tumbling is characterized by full in-plane rotations of the nematic director, whereas wagging just implies finite back-and-forth-motion in angular space.

To quantify the differences, we have calculated the angle $\vartheta=\arccos \left[\left(\mathbf{d}^{A}\right.\right.$. $\left.\mathbf{d}^{B}\right) /\left(\left|\mathbf{d}^{A}\right|\left|\mathbf{d}^{B}\right|\right)$ between the two nematic directors, $\mathbf{d}^{A}$ and $\mathbf{d}^{B}$. For in-plane motion, the Cartesian components of (un-normalized) $\mathbf{d}^{i}$ are related (via a linear transformation [69]) to the components $q_{0}^{i}, q_{1}^{i}, q_{2}^{i}$ of the full Q-tensors. Performing the scalar products between the directors then yields

$$
\vartheta(t)=\arccos \left(\frac{q_{j}^{A} q_{j}^{B}}{\sqrt{q_{j}^{A} q_{j}^{A}} \sqrt{q_{j}^{B} q_{j}^{B}}}\right) .
$$



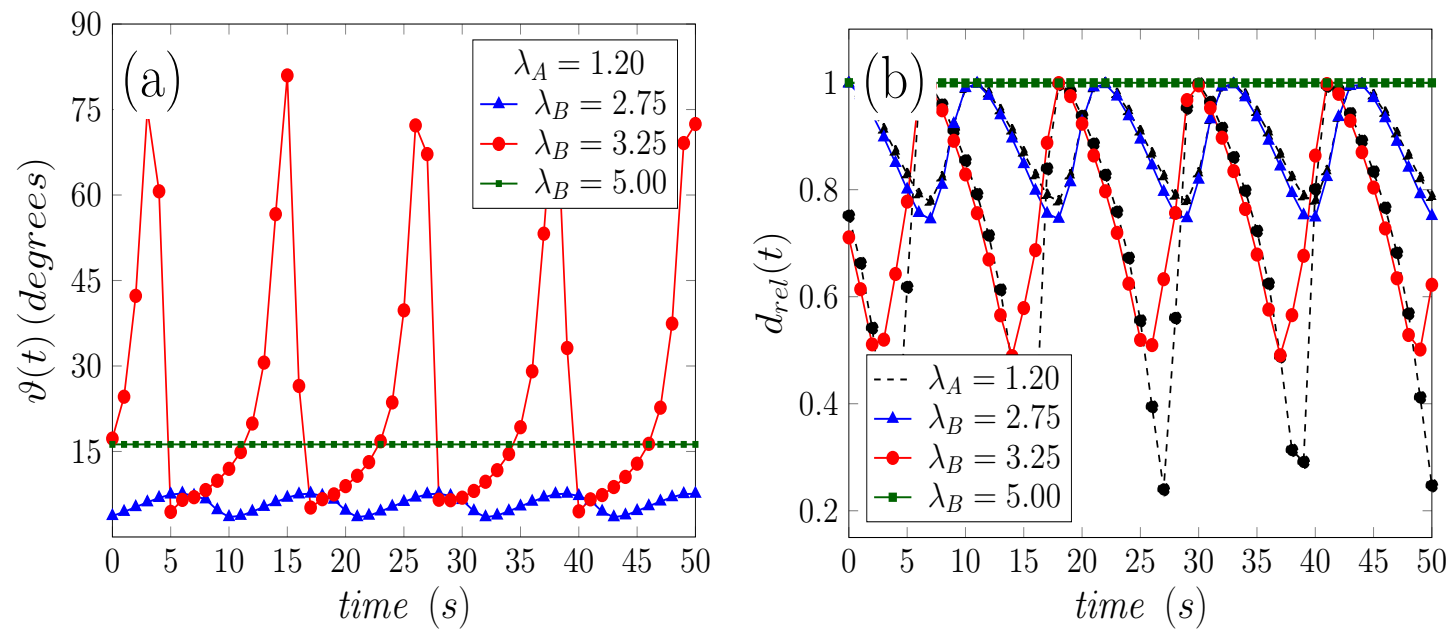

Figure 6. a) Angle between the nematic directors as function of time at $\dot{\gamma}=7.5$ and three values of $\lambda_{B}$. The green solid line ( $\left.\mathbf{\square}\right)$ corresponds to shear-aligned states of both species $\left(\lambda_{B}=5.0\right)$, whereas the blue line $(\boldsymbol{\Lambda})$ corresponds to synchronized tumbling states $\left(\lambda_{B}=2.75\right)$. Finally, the red line $(\bullet)$ represents a case where the A-species (B-species) is in a tumbling (wagging) state $\left(\lambda_{B}=3.75\right)$. b) Relative alignment of the nematic directors as a function of time at $\dot{\gamma}=7.5$. The dashed black lines and the solid lines correspond to the behavior of the A-species $\left(\lambda_{A}=1.20\right)$ and $\mathrm{B}-$ species (with varying $\lambda_{B}$ ), respectively. The $(\boldsymbol{\square})$ symbol correspond to shear-aligned states of both components $\left(\lambda_{B}=5.0\right)$ whereas the $(\boldsymbol{\Lambda})$ correspond to synchronized tumbling states $\left(\lambda_{B}=2.75\right)$. Finally, $(\bullet)$ represents the coexisting $\mathrm{T} / \mathrm{W}$ state $\left(\lambda_{B}=3.75\right)$.

where $j=0,1,2$ and we used the Einstein convention.

Numerical results at $\dot{\gamma}=7.5$ and three values of $\lambda_{B}$ are plotted in figure 6 (a), where we focus on times after the initial transient period. At the largest $\lambda_{B}$ considered, both species are in a shear-aligned state. For single-component systems, it is well known [35, 32, 68] that the shear-aligned state is characterized by a finite angle between the (stationary) director and shear direction, and that this "flow angle" depends on the tumbling parameter. Therefore, one would expect the two directors of the mixture to have different (stationary) flow angles with respect to the shear direction, and consequently, to enclose a finite angle in between. This is exactly what we see from the corresponding curve in figure 6 (a). At $\lambda_{B}=3.25$, both species are in the tumbling state (see figure 5), with the directors displaying full rotations. Still, we see from figure 6(a) that the angle between the tumbling directors is close to zero at all times considered. We interpret this behavior as (nearly) synchronous tumbling. At $\lambda_{B}=3.25$, however, the behavior is markedly different: Here we observe a periodic variation of the angle between the directors, reaching values up to nearly 90 degree. This reflects the simultaneous appearance of tumbling (species A) and wagging (species B).

To further compare the in-plane oscillations of the nematic directors in the different regions of the state diagram (see figure 4) we also look at their relative amplitudes. To this end we introduce a quantity that compares, for each component of the mixture, the instantaneous magnitude $\left|\mathbf{d}^{i}\right|(t)$ with its maximum value $\max \left\{\left|\mathbf{d}^{i}\right|\right\}$ (we recall at this 
point that the directors considered here are not normalized to one). In terms of the components $q_{0}^{i}, q_{1}^{i}, q_{2}^{i}$, the relative alignment is therefore given by

$$
d_{r e l}^{A(B)}(t)=\frac{\sqrt{q_{j}^{A(B)} q_{j}^{A(B)}}}{\max \left\{\sqrt{q_{j}^{A(B)} q_{j}^{A(B)}}\right\}},
$$

where $j=0,1,2$ and we used the Einstein convention. This quantity is shown in figure 6(b). As expected, in the shear-aligned state $\left(\lambda_{B}=5.0\right)$ the relative alignment of both components does not oscillate in time. Further, when both nematic directors are tumbling (i.e., perform full rotations, here at $\lambda_{B}=2.75$ ), the oscillations are in phase and also their magnitude is comparable. In contrast, in the coexisting $\mathrm{T} / \mathrm{W}$ state $\left(\lambda_{B}=3.25\right)$, there is a marked difference between the amplitude of oscillations. Still, the oscillations are in phase. Therefore, we conclude that both, the $\mathrm{T}$ state and the coexisting $\mathrm{T} / \mathrm{W}$ state are characterized by synchronous motion.

3.3.2. Variation of the cross coupling In view of the variety of dynamical states at fixed cross coupling parameter $\Theta_{A B}$ it is interesting to further explore the impact of this parameter. To this end we present in figure 7 the state diagrams obtained at fixed tumbling parameters $\lambda_{A}=1.20$ and $\lambda_{B}=3.0$, in the plane spanned by $\Theta_{A B}$ and $\dot{\gamma}$. At $\dot{\gamma}=0$ (equilibrium), all systems considered are in the (meta-)stable nematic phase.
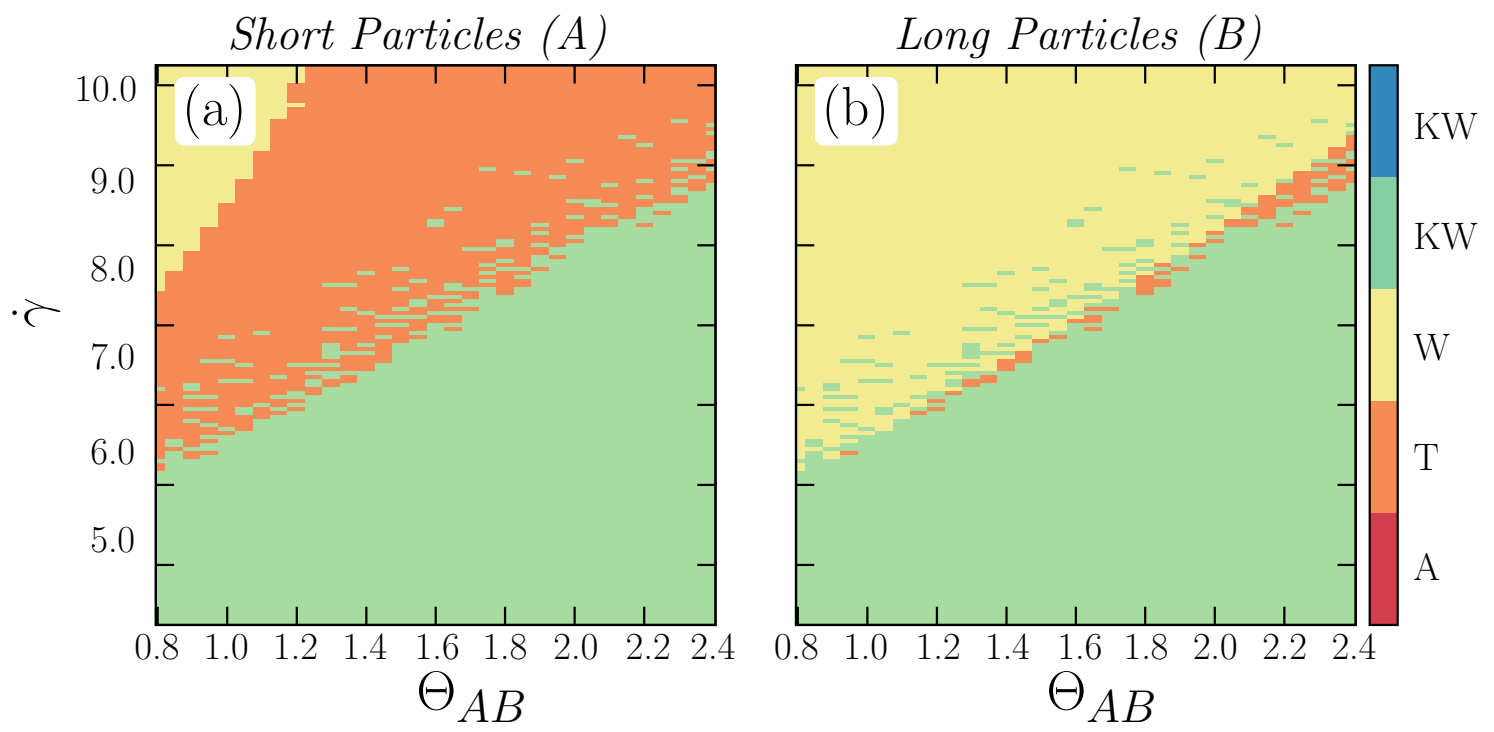

Figure 7. Dynamical state diagram in the plane spanned by shear rate and cross coupling parameter for a binary mixtures characterized by $\Theta_{A}=-0.25, \Theta_{B}=-0.25$, $\lambda_{A}=1.2$ and $\lambda_{B}=3.0$. Parts a) and b) illustrate the behavior of the short and long particles, respectively.

At small shear rates $\dot{\gamma} \lesssim 5$.0, both species display an out-of-plane KT state for all cross coupling parameters considered. At larger shear rates the out-of-plane oscillations then transform into in-plane oscillations of $\mathrm{W}$ or $\mathrm{T}$ type. As indicated from figure 7 , 
an increase of $\Theta_{A B}$ shifts this boundary between KT and $\mathrm{T}$ or $\mathrm{W}$ towards larger shear rates for both components of the mixture. A further effect becomes apparent when we consider a fixed, high shear rate, e.g., $\dot{\gamma}=8.0$. Here, an increase of $\Theta_{A B}$ can induce profound changes of the dynamical state, such as a change from $\mathrm{W}$ to $\mathrm{T}$ to KT. Along this way, there a large regions where the two components display different dynamical behavior (coexistence of $\mathrm{T}$ and $\mathrm{W}$ ), similar to what we have discussed before. Finally, we notice the small "islands" of KT regions within the T state (A-species) or W-state (B-species). This indicates a bi-stability of the solutions which may be interpreted as a coexistence of in-shear-plane and out-of-shear plane oscillations (as already mentioned in [34]). At this point, it would be very interesting to extend the present analysis to spatially inhomogeneous systems: Indeed, as previously shown for a one-component system [37, 71], bistability in the homogeneous system can translate to spatially separated dynamical coexistence of different states, when one allows for inhomogeneity.

\section{Concluding remarks}

In this paper we have combined a microscopic free energy density functional [43] and mesoscopic (continuum) equations to investigate the orientational dynamics of binary mixtures of rod-like particles under planar (Couette) shear flow. To establish the link between the microscopic and mesoscopic parts, we have performed an expansion of the (Parson-Lee) functional in powers of the $\mathrm{Q}$-tensors of each species. This yields a Landau-like expression for the orientational part of the free energy, the difference to the traditional LdG approach being that the coefficients depend on the details of the pair interaction such as the aspect ratio. The resulting functional enters via the relaxational term in the Doi-Hess equations for the $\mathbf{Q}$-tensors.

To explore the mixture's behavior in thermodynamic equilibrium (zero shear), we have presented several calculations focusing on the stability of isotropic and nematic states. Indeed, experience with one-component systems [34] shows that the nature of the underlying equilibrium state is crucial for the dynamical behavior under shear. The equilibrium mixtures considered here were characterized by particles with equal diameters and different length. This is somewhat in contrast to other recent studies focusing on "thick-thin" mixtures of particles with equal length and different diameters [2]. However, as noted at the end of section 2.4.2, the present approach can be easily modified towards thick-thin mixtures by a simple rescaling of densities. A more severe restriction of our equilibrium investigations is that we have concentrated on the orientational part of the free energy alone. Thus, our stability analysis does not give any information about phase transitions (or coupled phase transitions) involving the number densities as order parameters, an example being macroscopic demixing. This would clearly be an important extension of the present work.

As a second step, we have explored the shear-induced non-equilibrium behavior starting from a globally nematic state. Similar to one-component systems, we observe 
a variety of oscillatory dynamical states of the nematic directors, either with inplane or with out-of-plane symmetry, as well as steady states. One interesting new feature occurring for the binary mixture is the appearance of states where the two directors perform different oscillatory motion. In particular, we observe a simultaneous appearance of tumbling motion (short particles) and wagging motion (long particles). However, although the actual motion is different, the time-dependence of quantities such as the angle between the directors reflects that the oscillations are in synchrony.

Another interesting result is the strong dependence of the dynamical state diagrams on the coupling parameter $\Theta_{A B}$ determined by the interactions between different species. For example, by increasing $\Theta_{A B}$ at fixed shear rate, one can find transitions from inplane oscillations to out-of-plane oscillations (kayaking-tumbling), either of same or different type for the two components considered. In that sense, $\Theta_{A B}$ has a similarly important role as the tumbling parameter $\lambda$. This prediction may be particularly interesting for experiments.

Given the appearance of mixed ("coexisting") dynamical states, it would be very interesting to extend the present analysis to spatially inhomogeneous systems, thereby allowing for spatial domains characterized by different dynamical behavior. From the methodological point of view, a straightforward strategy to include inhomogeneity into the equilibrium part is to perform a gradient expansion of the mesoscopic free energy functional. Within the dynamical part, one should then take into account the feedback effect caused by inhomogeneous orientational order parameters on the velocity profile of the flow (see, e.g., [63]). A further route of research concerns the rheological behavior of the mixture and its "tunability", e.g. via the aspect ratios. Work in these directions is in progress.

\section{Acknowledgments}

We gratefully acknowledge financial support from the Deutsche Forschungsgemeinschaft (DFG) through the Research Training Group 1558, project B3.

\section{Appendix A. Coefficients of the excluded volume of hard spherocylinders}

The excluded volume of two spherocylinders, whose particle axes enclose the angle $\gamma$, is given in (18). In the following we aim at deriving analytic expressions for the coefficients

$V_{k}^{i j}, k=0,2,4$, appearing in the expansion of $V_{e x c}(\gamma)$ in Legendre polynomials [see equations (9) -(10)]. To this end we expand the functions $|\cos \gamma|$ and $E(\sin \gamma)$ in (18) up to fourth order in $\sin \gamma$, yielding

$$
\begin{aligned}
|\cos \gamma| & =(1-\sin \gamma)^{1 / 2} \\
& =1-\frac{1}{2} \sin ^{2} \gamma-\frac{1}{8} \sin ^{4} \gamma+\mathcal{O}(6),
\end{aligned}
$$

and

$$
E(\sin \gamma)=\frac{\pi}{2}-\frac{\pi}{8} \sin ^{2} \gamma-\frac{3 \pi}{128} \sin ^{4} \gamma+\mathcal{O}(6) .
$$


Neglecting the term $\mathcal{O}(6)$, the excluded volume becomes

$$
\begin{gathered}
V_{\text {exc }}^{O(4)}(\gamma)=\frac{\pi}{4} c_{1}+\frac{\pi}{4} c_{2} \sin \gamma+\frac{\pi}{4} c_{3}\left(1-\frac{1}{2} \sin ^{2} \gamma-\frac{1}{8} \sin ^{4} \gamma\right) \\
+c_{4}\left(\frac{\pi}{2}-\frac{\pi}{8} \sin ^{2} \gamma-\frac{3 \pi}{128} \sin ^{4} \gamma\right)+c_{5} \sin \gamma
\end{gathered}
$$

where the constants $c_{i}$ depend on the aspect ratio and diameters of the particles and are given by

$$
\begin{array}{ll}
c_{1}=\kappa_{i} d_{i}^{3}+\kappa_{j} d_{j}^{3}, & c_{2}=d_{i} d_{j}\left(d_{i}+d_{j}\right), \\
c_{3}=\kappa_{i} d_{i} d_{j}^{2}+\kappa_{j} d_{j} d_{i}^{2}, & c_{4}=\left(\kappa_{i} d_{i}+\kappa_{j} d_{j}\right) d_{i} d_{j} \\
c_{5}=\kappa_{i} \kappa_{j} d_{i} d_{j}\left(d_{i}+d_{j}\right) . &
\end{array}
$$

We note that the coefficients $c_{3}$ and $c_{4}$ vanish within the original Onsager approach [7].

Starting from (A.3), we can now employ (10) to calculate the expansion coefficients of interest $(k=0,2,4)$ :

$$
V_{k}^{i j}=\frac{2 k+1}{2} \int_{0}^{\pi} d \gamma V_{\text {exc }}^{O(4)}(\gamma) P_{k}(\cos \gamma) \sin \gamma .
$$

Using $P_{0}(\cos \gamma)=1$ and performing the angular integral, we obtain the zero-order coefficient

$$
V_{0}^{i j}=\frac{\pi}{4} c_{1}+\frac{\pi^{2}}{16} c_{2}+\frac{3 \pi}{20} c_{3}+\frac{97 \pi}{240} c_{4}+\frac{\pi}{4} c_{5} .
$$

To calculate $V_{2}^{i j}$ we use that $P_{2}(\cos \gamma)=\frac{1}{2}\left(3 \cos ^{2} \gamma-1\right)$. Since

$$
\int_{0}^{\pi} d \gamma P_{2}(\cos \gamma) \sin \gamma=0
$$

all linear functions of $\sin \gamma$ do not contribute to the coefficient. However, powers of $\sin \gamma$ do contribute since

$$
\begin{aligned}
& \int_{0}^{\pi} d \gamma \sin \gamma P_{2}(\cos \gamma) \sin \gamma=-\frac{\pi}{16} \\
& \int_{0}^{\pi} d \gamma \sin ^{2} \gamma P_{2}(\cos \gamma) \sin \gamma=-\frac{4}{15} \\
& \int_{0}^{\pi} d \gamma \sin ^{4} \gamma P_{2}(\cos \gamma) \sin \gamma=-\frac{32}{105}
\end{aligned}
$$

Inserting these results into (A.5) with $k=2$ we obtain

$$
V_{2}^{i j}=-\frac{5 \pi^{2}}{128} c_{2}+\frac{3 \pi}{28} c_{3}+\frac{17 \pi}{168} c_{4}-\frac{5 \pi}{32} c_{5} .
$$

The coefficients $V_{4}^{i j}$ involve the Legendre polynomial $P_{4}(\cos \gamma)=\frac{1}{8}\left(35 \cos ^{4} \gamma-30 \cos ^{2} \gamma+\right.$ $3)$. We find that integrals involving linear and cubic terms in $\sin \gamma$ vanish whereas the other integrals give non-zero results. Specifically,

$$
\begin{aligned}
& \int_{0}^{\pi} d \gamma \sin \gamma P_{4}(\cos \gamma) \sin \gamma=-\frac{\pi}{128} \\
& \int_{0}^{\pi} d \gamma \sin ^{4} \gamma P_{4}(\cos \gamma) \sin \gamma=\frac{16}{315} .
\end{aligned}
$$


With this we finally obtain

$$
V_{4}^{i j}=-\frac{9 \pi^{2}}{1024} c_{2}-\frac{\pi}{140} c_{3}-\frac{3 \pi}{560} c_{4}-\frac{9 \pi}{256} c_{5} .
$$

It is worth noting that, since the aspect ratio and the diameter are always positive, the coefficients $c_{i}$ must be positive as well. It follows that $V_{4}^{i j}$ is always negative, whereas

the sign of $V_{2}^{i j}$ depends on the combination of sizes. This is different from standard Onsager theory where $c_{3}=c_{4}=0$ and thus, $V_{2}^{i j}$ is always negative.

\section{References}

[1] van der Kooij R M and Lekkerkerker H N W 2000 Phys. Rev. Lett. 84(4) 781-784

[2] Purdy K R, Varga S, Galindo A, Jackson G and Fraden S 2005 Phys. Rev. Lett. 94

[3] Varga S, Purdy K, Galindo A, Fraden S and Jackson G 2005 Phys. Rev. E 72(5) 051704

[4] ten Brinke A J W, Bailey L, Lekkerkerker H N W and Maitland G C 2008 Soft Matter 4(2) $337-348$

[5] van Westen T, Vlugt T J H and Gross J 2014 J. Chem. Phys 140034504

[6] Woolston P and van Duijneveldt J S 2015 J. Chem. Phys 142184901

[7] Onsager L 1949 Ann. N. Y. Acad. Sci. 51

[8] Parsons J D 1979 Phys. Rev. A 19(3) 1225-1230

[9] Lee S D 1987 J. Chem. Phys 87 4972-4974

[10] Somoza A M and Tarazona P 1989 J. Chem. Phys 91 517-527

[11] Bolhuis P and Frenkel D 1997 J. Chem. Phys 106 666-687

[12] Cuetos A, Martinez-Haya B, Lago S and Rull L 2007 Phys. Rev. E 75

[13] Löwen H 2008 J. Phys. Condens. Matter 20404201

[14] Löwen H 2012 J. Phys. Condens. Matter 24460201

[15] Guu D, Dhont J and Lettinga M P 2013 Eur. Phys. J. ST 222 2739-2755

[16] Olmsted P D 1991 The effect of shear flow on the isotropic-nematic transition in liquid crystals (Ph.D. Thesis, University of Illinois, Urbana-Champaign)

[17] Dogic Z and Fraden S 2007 Phase behavior of rod-like viruses and virus-sphere mixtures (Wiley Verlag)

[18] Fielding S M and Olmsted P D 2003 Phys. Rev. E 68(3) 036313

[19] Fielding S M and Olmsted P D 2006 Phys. Rev. Lett. 96(10) 104502

[20] Olmsted P D 2008 Rheol. Acta 47 283-300

[21] Lettinga M P, Dogic Z, Wang H and Vermant J 2005 Langmuir 21 8048-8057

[22] Ripoll M, Holmqvist P, Winkler R G, Gompper G, Dhont J K G and Lettinga M P 2008 Phys. Rev. Lett. 101(16) 168302

[23] Tao Y G, den Otter W K and Briels W J 2009 Europhys. Lett. 8656005

[24] Ericksen J 1962 Arch. Rational Mech. Anal. 9 371-378

[25] Leslie F 1968 Arch. Rational Mech. Anal. 28 265-283

[26] Hess S 1975 Z. Naturforsch. 30a

[27] Hess S 1976 Z. Naturforsch. 31a

[28] Doi M and Edwards S 1978 J. Chem. Soc. 74

[29] Pardowitz I and Hess S 1980 Phys. A 100

[30] de Gennes P G 1993 The physics of liquid crystals (Clarendon Press, Oxford)

[31] Hess S 2015 Tensors for Physics (Springer Verlag)

[32] Rienäcker G and Hess S 1999 Phys. A 267

[33] Rienäcker G 2000 Orientational dynamics of nematic liquid crystals in a shear flow (Shaker, Verlag)

[34] Strehober D A, Engel H and Klapp S H L 2013 Phys. Rev. E 88 
[35] Rienäcker G, Kröger M and Hess S 2002 Phys. Rev. E 66

[36] Heidenreich S, Ilg P and Hess S 2006 Phys. Rev. E 73(6) 061710

[37] Das M, Chakrabarti B, Dasgupta C, Ramaswamy S and Sood A K 2005 Phys. Rev. E 71(2) 021707

[38] Evans R 1979 Adv. Phys. 28 143-200

[39] Evans R 1992 Density functionals in the theory of nonuniform fluids (Marcel Dekker, New York)

[40] Tarazona P, Cuesta J A and Martínez-Ratón Y 2008 Density Functional of Hard Particle Systems vol 753 (Springer Verlag)

[41] Singh S 2000 Phys. Rep. $324107-269$

[42] Vink R L C, Wolfsheimer S and Schilling T 2005 J. Chem. Phys 123074901

[43] Malijevsky A, Jackson G and Varga S 2008 J. Chem. Phys 129

[44] Wittkowski R, Löwen H and Brand H R 2010 Phys. Rev. E 82(3) 031708

[45] Menzel A M 2015 Phys. Rep. $5541-45$

[46] Zhou X, Chen H and Iwamoto M 2004 J. Chem. Phys 1201832

[47] Kröger M, Ammar A and Chinesta F 2008 J Nonnewton Fluid Mech 149

[48] Grandner S, Heidenreich S, Hess S and Klapp S H L 2007 Eur. Phys. J. E Soft Matter 24

[49] McCourt F, Beenakkeran J, Koehler W and Kuscer I 1991 Nonequilibrium phenomena in polyatomic gases (Clarendon Press, Oxford)

[50] Vroege G J and Lekkerkerker H N W 1992 Rep. Prog. Phys. 551241

[51] Gray C G and Gubbins K E 1984 Theory of molecular liquids (Clarendon Press, Oxford)

[52] Kröger M 2003 Phys. Rep. 390

[53] Mulder B M 2005 Mol. Phys. 103

[54] Carnahan N F and Starling K E 1969 J. Chem. Phys 51 635-636

[55] Camp P J and Allen M P 1996 Phys. A 229 410-427

[56] Wensink H H, Vroege G J and Lekkerkerker H N W 2001 J. Chem. Phys 115 7319-7329

[57] Frenkel D 1989 Statistical mechanics of liquid crystals (Les Houches session LI)

[58] Dingemans T, Madsen L, Zafiropoulos N, Wenbin L and Samulski E 2006 Phil. Trans. R. Soc. A $3642681-2696$

[59] Solomon M J and Spicer P T 2010 Soft Matter 6

[60] Mewis J and Wagner N 2012 Colloidal Suspension Rheology (Cambridge)

[61] Dennison M, Dijkstra M and van Roij R 2011 Phys. Rev. Lett. 106

[62] Courant R and John F 1989 Introduction to calculus and analysis (Springer Verlag)

[63] Heidenreich S 2009 Orientational Dynamics and Flow Properties of Polar and Non-Polar Hard-Rod Fluids (PhD Thesis, TU-Berlin)

[64] Purdy K R, Dogic Z, Fraden S, Rühm A, Lurio L and Mochrie S G J 2003 Phys. Rev. E 67031708

[65] Hess S and Kröger M 2004 J. Phys. Condens. Matter 16

[66] Hess S and Kröger M 2005 in Computer Simulations of Liquid Crystals and Polymers (Springer Verlag) pp 295-333

[67] Press W H, Teukolsky S A, Vetterling W T and Flannery B P 1992 Numerical Recipes in C (2Nd Ed.): The Art of Scientific Computing (New York, NY, USA: Cambridge University Press) ISBN 0-521-43108-5

[68] Hess O and Hess S 1994 Physica A 207

[69] Klapp S H L and Hess S 2010 Phys. Rev. E 81(5) 051711

[70] Cates M E, Head D A and Ajdari A 2002 Phys. Rev. E 66025202

[71] Chakraborty D, Dasgupta C and Sood A K 2010 Phys. Rev. E 82(6) 065301 